\title{
BPS and non-BPS states in a supersymmetric Landau-Ginzburg theory
}

\author{
Alin Tirziu and Paul Fendley \\ Department of Physics, University of Virginia \\ Charlottesville, VA 22904-4714
}

October 24, 2018

\begin{abstract}
We analyze the spectrum of the $\mathcal{N}=(2,2)$ supersymmetric Landau-Ginzburg theory in two dimensions with superpotential $W=X^{n+2}-\lambda X^{2}$. We find the full BPS spectrum of this theory by exploiting the direct connection between the UV and IR limits of the theory. The computation utilizes results from the Picard-Lefschetz theory of singularities and its extension to boundary singularities. The additional fact that this theory is integrable requires that the BPS states do not close under scattering. This observation fixes the masses of non-BPS states as well.
\end{abstract}

\section{Introduction}

Supersymmetric field theories have many remarkable properties. In many cases, special quantities like the superpotential are holomorphic functions of the fields. The holomorphy makes it possible to obtain exact information about the theory. In many different types of theories, one can compute the exact spectrum of certain particles known as BPS states [1]. Probably the most famous such computation is for four-dimensional $\mathcal{N}=2$ supersymmetric gauge theories, where Seiberg and Witten showed how to compute the BPS spectrum by exploiting the holomorphy of the superpotential and the fact that its singularity structure and monodromies can be inferred by perturbative arguments [2].

Before the work of [2], a very close analog of this computation had already been done in $\mathcal{N}=(2,2)$ supersymmetric field theories in $1+1$ dimensions. It was shown in [3] how one could derive a differential equation whose solution gives directly the exact BPS mass spectrum. The computation utilizes a technique called topological-antitopological fusion, introduced in $\llbracket 4$. This work was extended further in [5], where it was shown how to extract the BPS spectrum without having to find the full solution of the differential equation. Instead, the BPS masses, a property of the infrared, are related directly the charges of the fields in the Ramond sector at the ultraviolet conformal point.

For a variety of reasons, it seemed to us worthwhile to revisit these techniques. There has renewed interest in closely related two-dimensional theories with supersymmetry in the last few years (see e.g. [6, 7, 8]). It is quite remarkable that exact non-perturbative information can be obtained in a strongly-interacting field theory, and in our opinion, the results of [4, 3, 5] have 
not yet been fully exploited. Most of the examples studied in these earlier papers were fairly well understood prior to that work. In particular, many of them correspond to integrable field theories, for which there exist a number of standard techniques for deriving exact information. By using $S$-matrix techniques, the exact spectrum for many $\mathcal{N}=(2,2)$ theories had already been found in [9, 10, 11]. Thus it would be useful to apply the results of [3, 田, 泡 to cases where the answer is not known already. Moreover, even in the known cases, computing the spectrum is done indirectly - one makes an ansatz and checks its consistency by the powerful constraints of integrability. The methods of [5] allow a direct and exact computation of the BPS spectrum.

The key result exploited in this paper is quite simple to understand. The BPS states here form doublets under the supersymmetry, while all other states form a quartet. In $\mathcal{N}=(2,2)$ theories, there is a $U(1)$ fermion-number symmetry, denoted by $F$. The supercharges have fermion number \pm 1 , so the particles in the BPS doublet have fermion numbers $(f, f-1)$ for some number $f$, while a quartet has charge $(f+1, f, f, f-1)$. The quantity introduced in [3] is a thermodynamic trace,

$$
C \propto \operatorname{tr}\left[F(-1)^{F} e^{-\beta H}\right]
$$

where $\beta$ the inverse temperature and $H$ the Hamiltonian on open space. The key property of $C$ is that only the BPS states appear in the one-particle contributions to this trace (the leading contributions at $\beta$ large). A BPS doublet of mass $m$ results in a one-particle contribution proportional to

$$
f(-1)^{f}+(f-1)(-1)^{f-1}=(-1)^{f}
$$

to the trace. However, the quartet one-particle contribution vanishes, because $(f+1)-f-$ $f+(f-1)=0$. As we will discuss, the fermion number $f$ and the mass $m$ of the BPS states are known from the superpotential. Thus computing $C$ with a given set of boundary conditions gives the exact multiplicities of any BPS states obeying these boundary conditions. In fact, in [5], it was shown how to find the large- $m$ behavior of $C$ (and hence the multiplicities) without having to compute the whole thing.

The theories studied here correspond to superconformal minimal models perturbed by a relevant operator. In terms of a Landau-Ginzburg superfield $X$, the superpotential is $X^{n+2}-$ $\lambda X^{2}$. In this paper, we derive the exact BPS spectrum of these models, and use the integrability to learn about the non-BPS states. In a sequel [12], we will give their $S$-matrix. The series of models studied here is one of three sets of integrable $\mathcal{N}=(2,2)$ theories found by deforming the superconformal minimal model into a massive field theory [9, 13, 14]. The other two deformations are well understood [10, 11]. The theory here is more complicated, and the $S$-matrix approach ran aground because of technical complications [15]. The differential equations arising from topological-antitopological fusion were found in [迎, but their general asymptotic behavior is not known, and so the BPS spectrum was not extracted. The spectrum has been guessed at [13, 16], but no authoritative results were known. Another reason for doing our computation is that we will find some very interesting behavior. Due to the integrability, we can not only infer the masses of non-BPS states, but prove that they can be produced in a scattering event involving only the BPS states.

In section 2 we review the main characteristics of supersymmetric solitons in two dimensions, and discuss the theories with superpotential $W=X^{n+2}-\lambda X^{2}$. In section 3 we review the work of [3, 5]. In particular we discuss the index $C$, and the relation between the BPS spectrum and the charges of the chiral fields. The proof and further use of this relation is directly related to singularity theory: the BPS spectrum is encoded in the monodromy matrix of the vanishing cycles. We recall, as needed, main results from the Picard-Lefschetz theory of singularities of differentiable maps [17, 18]. The computation for $n$ odd in section 4 is a straightforward 
application of the above relation. The computation of BPS spectrum for $n$ even in section 5 requires additional mathematical results, from the singularity theory of functions on manifolds with boundary. In section 6 we prove the existence of non-BPS states in this theory, and present our conclusions.

\section{Supersymmetric solitons in $1+1$ dimensions}

In this section we review $\mathcal{N}=(2,2)$ supersymmetric solitons in $1+1$ dimensions, and introduce the theories studied in this paper.

The two left supersymmetry operators are denoted $Q^{+}, Q^{-}$, and the two right are denoted $\bar{Q}^{+}, \bar{Q}^{-}$. There is a global $U(1)$ fermion-number symmetry called, generated by $F$. We take space $\sigma$ to be the real line. When there are multiple vacua in a theory, there exist field configurations which are in one vacuum $a$ at $\sigma=-\infty$ and in another vacuum $b$ at the other end $\sigma=+\infty$. These configurations are known as solitons, or in $1+1$ dimensions, kinks. In the sector $a b$, the superalgebra is

$$
\begin{array}{ll}
\left\{Q^{+}, Q^{-}\right\}=H+P & \left\{\bar{Q}^{+}, \bar{Q}^{-}\right\}=H-P \\
\left\{Q^{+}, \bar{Q}^{+}\right\}=\Delta & \left\{Q^{-}, \bar{Q}^{-}\right\}=\bar{\Delta} \\
{\left[F, Q^{ \pm}\right]= \pm Q^{ \pm}} & {\left[F, \bar{Q}^{ \pm}\right]=\mp \bar{Q}^{ \pm}}
\end{array}
$$

where $H$ and $P$ are the Hamiltonian and the momentum operators, and all other anticommutators vanish. The appearance of a central term $\Delta_{a b}$ in superalgebras on non-compact spaces was noted long ago [19]. An important consequence of the central term is a lower bound on the mass of any configuration in a given sector $a b$, namely [1, 19]

$$
m_{a b} \geqslant\left|\Delta_{a b}\right| \text {. }
$$

The states whose mass saturates this bound are called BPS states. In an $\mathcal{N}=(2,2)$ theory in $1+1$ dimensions, the BPS states have another very special property: they and only they form two-dimensional multiplets under the supersymmetry algebra. Other states form irreducible four-dimensional representations.

Many supersymmetric theories have an elegant Landau-Ginzburg description [20, 21]. An $\mathcal{N}=(2,2)$ Landau-Ginzburg theory is defined in terms of a set of $N$ chiral superfields $\phi^{i}$ each consisting of a complex boson and a Dirac fermion. The action is

$$
S=\frac{1}{2} \int d^{2} z d^{4} \theta K\left(\phi^{i}, \bar{\phi}^{i}\right)+\int d^{2} z d \theta^{+} d \theta^{-} W\left(\phi^{i}\right)+h . c .
$$

where $W$, the superpotential, is a holomorphic function and $K$ is a Kähler potential. The bosonic part of the LG action is given by:

$$
S=\frac{1}{2} \int d^{2} z\left(g_{i \bar{j}} \partial_{\mu} X^{i} \partial_{\mu} \bar{X}^{j}+g^{i \bar{j}} \partial_{i} W \overline{\partial_{j} W}\right)
$$

where $X^{i}$ is the bosonic part of $\phi^{i}$ and $g_{i \bar{j}}=\partial_{i} \bar{\partial}_{j} K$ is a positive-definite metric. Note the different kinds of derivatives: $\partial_{\mu}$ is the usual space-time derivative, whereas $\partial_{i}$ is a derivative with respect to the superfield $X^{i}$. The vacua $\left\{a_{k}\right\}_{k=1, \ldots, p}$ of the theory are obtained by minimizing the superpotential:

$$
\left.\frac{\partial W}{\partial X^{i}}\right|_{a_{k}}=0 \quad \text { for all } i=1 \ldots N
$$


The superpotential does not renormalize in a $\mathcal{N}=(2,2)$ theory in two dimensions. Moreover, by dimensional analysis, it follows that the Kähler term is always irrelevant if $X$ has non-zero dimension. This means that critical points can be described uniquely by their superpotential. One description of the superconformal minimal models is by a single superfield $X$ with $W=$ $X^{n+2}$ 20, 21]. This is called the $A$ series; for $n$ even, one can orbifold by the $X \rightarrow-X$ symmetry and obtain the $D$ series. The $D$ superconformal series can be described by two superfields $X$ and $Y$ with superpotential $W=X^{k}-X Y^{2}$.

We study time-independent solitons $X^{i}=X^{i}(\sigma)$, with $X^{i}(\sigma=-\infty)=a^{i}$ and $X^{i}(\sigma=\infty)=$ $b^{i}$. The mass of such a configuration is

$$
\begin{aligned}
m_{a b} & =\frac{1}{2} \int_{-\infty}^{\infty} d \sigma\left(g_{i \bar{j}} \partial_{\sigma} X^{i} \partial_{\sigma} \bar{X}^{j}+g^{i \bar{j}} \partial_{i} W \overline{\partial_{j} W}\right) \\
& =\frac{1}{2} \int_{-\infty}^{\infty} d \sigma\left|\partial_{\sigma} X^{i}-\omega g^{i \bar{j}} \overline{\partial_{j} W}\right|^{2}+\operatorname{Re}\left(\omega^{*} \Delta W_{a b}\right)
\end{aligned}
$$

where $\Delta W_{a b}=W(b)-W(a)$, and $\omega$ is an arbitrary phase. Since the metric is positive-definite, the first term in (3) is always greater than zero. Choosing $\omega=\Delta W /|\Delta W|$ yields the mass bound (2) with $\Delta_{a b}=\Delta W_{a b}$. The BPS solitons are those which saturate this bound:

$$
m_{a b}=\left|\Delta W_{a b}\right| .
$$

This computation is classical, but the crucial point is that the superpotential $W$ does not renormalize. Thus the exact non-perturbative mass of a BPS soliton is given by (田). From (3) we see that a BPS soliton must obey

$$
\partial_{\sigma} X^{i}=\omega g^{i \bar{j}} \overline{\partial_{j} W}
$$

As a consequence, the superpotential for each soliton trajectory obeys $\partial_{\sigma} W=\partial_{i} W \partial_{\sigma} X^{i}=$ $\omega|\partial W|^{2}$. This has a constant phase, so each BPS solution can be represented in $W$-plane as a straight line. Moreover, the mass is the length of the line.

In this paper we find the BPS spectrum of the Landau-Ginzburg theory with superpotential

$$
W(X)=\frac{X^{n+2}}{n+2}-\lambda \frac{X^{2}}{2} .
$$

For $\lambda=0$ this theory is the superconformal $A_{n+1}$ minimal series 20, 21]. For $\lambda \neq 0$ the relevant perturbation $X^{2}$ produces an integrable massive theory [13, 14]. The non-degenerate vacua are obtained by minimizing the superpotential $W(X)$, and in $X$-plane they are at the corners of an $n$-polygon and the origin:

$$
X_{0}=0 ; \quad X_{k}=\lambda^{1 / n} \exp \left(\frac{2 \pi i}{n} k\right)
$$

where $k=1 \ldots n$. In $W$-plane the vacua are:

$$
W_{0}=0, \quad W_{k}=-\frac{n}{2(n+2)} \lambda^{1+2 / n} \exp \left(\frac{4 \pi i}{n} k\right)
$$

They are at the corners of an $n$-polygon and the origin for $n$ odd, and at the corners of an $n / 2$-polygon and the origin for $n$ even. Because there is a $\mathbb{Z}_{n}$-symmetry acting transitively on 
the vacua in the $X$-plane, the soliton numbers between any two vacua $X_{i}, X_{j}$ except the origin depend only on $(i-j) \bmod n$ :

$$
\mu_{i j}=\mu(j-i), \quad i \neq j \neq 0
$$

with $\mu(i-n)=\mu(i)$. The soliton numbers connecting the vacuum $X_{0}$ to $X_{k}$ are all equal due to the same $\mathbb{Z}_{n}$-symmetry. We denote this number by $\mu_{0 k} \equiv \mu(0)$. There are thus $\left[\frac{n}{2}\right]+1$ distinct soliton numbers to be computed, namely $\mu(0), \mu(1), \ldots \mu\left(\left[\frac{n}{2}\right]\right)$.

Another interesting feature of these BPS solitons is that their fermion number can be fractional, even though the supercharges have integer charge [22]. The fractional part of the fermion number $f_{a b}$ in the Landau-Ginzburg description is [11]:

$$
e^{2 \pi i f_{a b}}=\text { phase }\left[\frac{\operatorname{det} H(b)}{\operatorname{det} H(a)}\right]
$$

where $H_{i j}=\partial_{i} \partial_{j} W$. In the theory (6), the states connecting the origin to another vacuum have fractional fermion number $\pm 1 / 2$. States connecting any two vacua except the origin have zero fractional fermion number, so these doublets consists of a boson and a fermion.

\section{Computing the BPS spectrum using Picard-Lefschetz theory}

The multiplicities and the masses of all BPS doublets in an $\mathcal{N}=(2,2)$ supersymmetric theory can be computed directly and exactly. In this section we review the method, and set up the problem to be solved in the remainder of the paper.

The Witten index [23] $\operatorname{Tr}(-1)^{F} \exp (-\beta H)$ on closed space counts the vacua of the theory, and is independent of deformations of the theory. In [3], an open-space generalization of this was introduced:

$$
C_{a b}=\lim _{L \rightarrow \infty} \frac{i \beta}{2 L} \operatorname{Tr}_{a b}\left[(-1)^{F} F e^{-\beta H}\right] .
$$

where $L$ is the length of space. The trace is over all states with boundary condition $a$ on the left and $b$ on the right. Unlike the Witten index, $C_{a b}$ does depend on $\beta$. It is an index in the sense that it does not depend on most deformations of theory; in a Landau-Ginzburg theory, it depends on the superpotential $W$, but not the Kähler term $K$.

The index $C_{a b}$ has simple expressions in both the ultraviolet and infrared limits of the theory. The leading contribution in the infrared limit $\beta \gg 1$ are the one-particle states, since multi-particle states have in general higher energy. As discussed in the introduction, only the two-dimensional representation of the supersymmetry algebra thus contributes to the index in this limit. In other words,

$$
\left.C_{a b}\right|_{\beta \rightarrow \infty}=-\frac{i \beta}{2 \pi} \mu_{a b} m_{a b} K_{1}\left(m_{a b} \beta\right)
$$

where $\mu_{a b}$ is a real anti-symmetric matrix counting the number of BPS states between two vacua $a$ and $b$. Thus if $C_{a b}$ can be computed, one can read off the soliton numbers of all BPS states. This equation is a slight over-simplification, because there are special cases where twoparticle states can also contribute to the index; these will be discussed below in section 5.2. The ultraviolet limit $\beta \rightarrow 0$ corresponds to studying the theory at the conformal point. In a massless theory, both left-moving and right-moving fermion numbers are conserved individually. In this limit, $C_{a b}$ gives the left (or right) charges of the Ramond vacua [3]:

$$
\left.C_{a b}\right|_{\beta \rightarrow 0}=q_{a b}^{R}
$$


These charges can easily be determined from the Landau-Ginzburg superpotential [20, 21]. For example, for the superconformal minimal models with superpotential $X^{n+2}$, the charges are

$$
q_{m}^{R}=\frac{1}{2}\left(1-\frac{2 m}{n+2}\right), \quad m=1 \ldots n+1
$$

We will discuss in detail below how the $n+1$ Ramond charges are related to the different possible boundary conditions $(a b)$ on the solitons.

Using the technique of topological-antitopological fusion developed in [4], it was shown in [3] that one can derive differential equations for the $C_{a b}$ in any $\mathcal{N}=(2,2)$ supersymmetric theory. In a Landau-Ginzburg theory, the superpotential gives these differential equations uniquely. The Ramond charges serve as boundary conditions to determine the solution. One can extract the soliton numbers by solving these differential equations, but as discussed in detail in [3], there is far more information in such a solution than just the soliton numbers. Moreover, the differential equations are non-linear, making the determination of asymptotic behavior (much less finding the entire solution) a non-trivial task. To avoid this complication, it was shown in [5] how to determine the $\beta \rightarrow \infty$ of the index directly, without having to find the full solution. This has been proved for any $\mathcal{N}=(2,2)$ supersymmetric theory in two dimensions by using an integral formulation of topological anti-topological fusion equations. For Landau-Ginzburg theories the connection has been proved by using the Picard-Lefschetz theory of singularities of differentiable maps.

The problem is to compute the number of BPS soliton doublets between any pair of vacua $a$ and $b$. BPS solitons satisfy the equation (5). The idea is to study solutions of this equation near all the critical points, and continuously deform them away from the critical points. If two such solutions meet at some point in the $W$-plane along the line between two vacua, then there is a soliton. This must be a consistent solution because the equation (5) is of first order.

More precisely, we assume all critical points of $W$ are non-degenerate $\left(\operatorname{det}\left(H_{i j}\right) \neq 0\right.$ at the critical points) and all critical values are distinct. By the Morse lemma there is local system of coordinates in the neighborhood of any critical point $a_{i}$ such that:

$$
W\left(X^{1}, \ldots, X^{N}\right)=W\left(a_{i}\right)+\sum_{i=1}^{N}\left(X^{i}\right)^{2}
$$

Without loss of generality one can take vacuum $a$ at the origin in $X$-plane with $W(a)=0$ and vacuum $b$ such that $W(b)$ is a real positive number. This means that $\omega=1$ for solutions of (3) near $a$. Also, up to second order terms the metric can be chosen to be $g_{i \bar{j}}=2 \delta_{i \bar{j}}$. The solution of equation (5) which as $\sigma=-\infty$ is at $a$ is:

$$
X^{i}=\lambda^{i} e^{\sigma}
$$

where the $\lambda^{i}$ and hence the $X^{i}$ near $a$ must be real. The superpotential of any soliton solution is thus a real $N-1$-dimensional sphere with radius denoted by $\delta$. The $\lambda^{i}$ and hence the radius $\delta$ vanish right at the critical point $a$. This sphere comprises all possible soliton solutions near $a$, and we call it the wavefront $\Delta_{a}$ :

$$
\Delta_{a}: \quad \sum_{i=1}^{N}\left(X^{i}\right)^{2}=\delta
$$

Not for all $\lambda^{i}$ there is a soliton solution: one must match the wavefront near $a$ to one near $b$. The wavefront near $b$ is defined analogously; all solutions of (5) near $b$ (here $\omega=-1$ ) form an $N$-1-dimensional sphere $\Delta_{b}$ which vanishes at $b$. 
These definitions of $\Delta_{a}, \Delta_{b}$ are exactly the definitions of vanishing cycles in singularity theory. We thus need to review some basics facts about the Picard-Lefschetz theory of singularities of differentiable maps [18]. Assume a holomorphic function $W: \mathbb{C}^{N} \rightarrow \mathbb{C}$ has only non-degenerate critical points $a_{1}, \ldots, a_{p}$ whose critical values are all distinct $W_{1}, \ldots, W_{p}$. If $t$ is a non-critical value in $W$-plane, the pre-image of it under $W$ is an $N-1$ complex dimensional space (non-singular level manifold) $V_{t} \equiv W^{-1}(t)$. One defines a system of non-self-intersecting paths $\left\{\gamma_{i}\right\}_{i=1, \ldots, p}$ to connect $t$ to each of the critical values $W_{i}: \gamma_{i}:[0,1] \rightarrow \mathbb{C}$ with $\gamma_{i}(u=0)=W_{i}, \gamma_{i}(u=1)=t$, $u \in[0,1]$. These paths do not cross any critical values. Also, they do not have to be cyclically ordered (weakly distinguished paths). For $u$ near zero one fixes in the level manifold $V_{\gamma(u)}$ an $N-1$ dimensional sphere: $S(u)=\sqrt{\gamma(u)-W_{i}} S^{N-1}$, where $S^{N-1}$ is the standard $N-1$ dimensional sphere in a system of local coordinates near a critical point $a_{i}$ :

$$
S^{N-1}=\left\{\left(X^{1}, \ldots, X^{N}\right): \sum_{i=1}^{N}\left(X^{i}\right)^{2}=1 ; \operatorname{Im} X^{j}=0 \text { for all } j=1, . ., N\right\}
$$

For $u=0$, the sphere $S(0)$ vanishes. Moving along the path $\gamma$ by homotopy lifting defines a family of $N-1$ dimensional cycles $S(u) \subset V_{\gamma(u)}$ diffeomorphic to spheres for all $u \in(0,1]$. Considering all critical points one obtains a set of $p$ vanishing cycles $\Delta_{i}$ represented by the spheres $S_{i}(1)$. These cycles are in the non-singular level set $V_{t}$ and they form a basis in the homology group $H_{N-1}\left(V_{t}\right)$. Each homology class $\Delta_{i}$ is defined modulo orientation. The nonsingular set $V_{t}$ is homotopy equivalent to a bouquet of $p$ spheres each having a real dimension $N-1$. When $t$ tends to one of the critical values $W_{i}$ the corresponding cycle $\Delta_{i}$ vanishes. In $V_{t}$ the cycles can intersect. The intersection number of two cycles (denoted by $\circ$ ) is a bilinear operation defined on the homology group in accord with the preferred orientation on $V_{t}$. The orientation on $\mathbb{C}^{N}$ is defined by the positive orientation on $\mathbb{R}^{2 N}$. For non-degenerate critical points with distinct critical values the intersection number takes values in the group of integers. In general the intersection number can take values in a subgroup of $\mathbb{C}$. By comparing the two preceding paragraphs one sees that the definition of cycles in singularity theory is indeed identical to that of the solutions of (5) near a critical point.

The result of [5] relates the number of soliton solutions between vacua $a$ and $b$ to the intersection number $\Delta_{a} \circ \Delta_{b}$ of the corresponding cycles. We pick an arbitrary point $t$ on the line between $W(a)$ and $W(b)$, and define the paths $\gamma_{a}, \gamma_{b}$ as straight lines connecting $t$ to $W_{a}$ and $W_{b}$ respectively. The two wavefronts $\Delta_{a}, \Delta_{b}$ are $N-1$ dimensional real cycles in $V_{t}$ for any $t$. In $W^{-1}(t)$ the two vanishing cycles $\Delta_{a}, \Delta_{b}$ intersect at a discrete number of points. The fact that equation (5) is of first order assures that for each intersection point the flow with the vector field defined by (5) reaches the critical points $a$ and $b$. Therefore for each intersection point of vanishing cycles we get a soliton solution. However, the number of points at which $\Delta_{a}$ and $\Delta_{b}$ intersect is not necessarily $\Delta_{a} \circ \Delta_{b}$, because the latter counts each intersection point with \pm 1 depending on the orientations. Moreover, as suggested by the supersymmetric index $C_{a b}$, the counting of BPS solitons is naturally done with the insertion $(-1)^{F} F$. Assume we have two different trajectories between vacua $a$ and $b$. Then, one has to compare the relative contribution of the two trajectories to $\Delta_{a} \circ \Delta_{b}$ and $(-1)^{F} F$. It was shown in [5] that if the two trajectories correspond to the same sign for intersection between cycles they also have the same fermion number mod 2, and if they have opposite intersection number their fermion numbers differ by 1 mod 2. Also, including another vacuum $c$ the relative signs in the sectors $(a c),(c b)$ and $(a b)$ are correlated with $\Delta_{a} \circ \Delta_{c}, \Delta_{c} \circ \Delta_{b}$ and $\Delta_{a} \circ \Delta_{b}$. Therefore the proper definition for the soliton number is [5]:

$$
\mu_{a b}=\sum_{(a b)} F(-1)^{F}=\Delta_{a} \circ \Delta_{b}
$$


where the sum is over all solitons in sector $(a b)$. This definition is valid for even number of fields. Because the intersection matrix of vanishing cycles for even number of fields is antisymmetric the above definition is consistent with the physical requirement that the matrix $\mu_{a b}$ be anti-symmetric. For an odd number of fields the soliton numbers are

$$
\mu_{a b}=\Delta_{a} \circ \Delta_{b} \quad \text { for } \quad a>b, \quad \mu_{a b}=-\Delta_{a} \circ \Delta_{b} \quad \text { for } \quad a<b
$$

Since $\left|\mu_{a b}\right|$ counts the number of BPS doublets, $\left|\Delta_{a} \circ \Delta_{b}\right|$ does as well.

The results of [5] thus reduce the problem of finding the BPS spectrum to that of calculating the intersection numbers of the cycles. This can be done by studying the behavior of the cycles under deformation of the paths $\gamma_{i}$. If the paths are continuously deformed the vanishing cycles do not change as long as the paths do not cross any critical value. But if during deformations a path crosses a critical value a change of basis appears. Let us consider two critical values $W_{i}$ and $W_{j}$ and two sets of paths $\gamma_{i}, \gamma_{j}$ and $\gamma_{i}^{\prime}, \gamma_{j}^{\prime}$ as in Figure 1. While $\gamma_{j}$ can be deformed

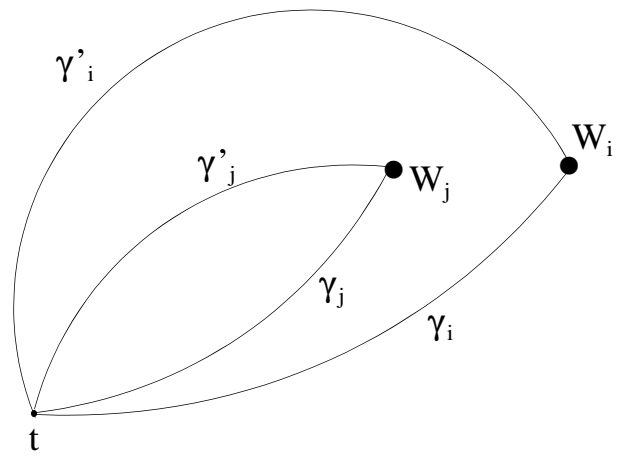

Figure 1: Paths $\gamma_{i}, \gamma_{j}$ change into $\gamma_{i}^{\prime}, \gamma_{j}^{\prime}$

into $\gamma_{j}^{\prime}$ without passing a critical value, the same is not true when deforming $\gamma_{i}$ into $\gamma_{i}^{\prime}$. This change of paths can be viewed as an operation denoted by $\alpha_{j}(i)$ described as: $\gamma_{i} \rightarrow \gamma_{i}^{\prime \prime}=\gamma_{i} \tau_{j}$ and $\gamma_{j} \rightarrow \gamma_{j}^{\prime \prime}=\gamma_{j}$, followed by a continuous change of paths: $\gamma_{i}^{\prime \prime} \rightarrow \gamma_{i}^{\prime}$ and $\gamma_{j}^{\prime \prime} \rightarrow \gamma_{j}^{\prime}$, where the simple loop $\tau_{j}$ is defined as the loop going along the path $\gamma_{j}$ from the point $t$ to the point $W_{j}$, going around the point $W_{j}$ in the positive direction (anticlockwise) and returning along the path $\gamma_{j}$ to the point $t$ (see Figure 2). The inverse of operation $\alpha_{j}(i)$ is the operation $\beta_{j}(i)=\gamma_{i} \tau_{j}^{-1}$. A result of Picard-Lefschetz theory is that the basis of vanishing cycles changes under the operation $\alpha_{j}(i)$ as:

$$
\begin{gathered}
\Delta_{j} \rightarrow \Delta_{j}^{\prime}=\Delta_{j} \\
\Delta_{i} \rightarrow \Delta_{i}^{\prime}=\Delta_{i}+(-1)^{N(N+1) / 2}\left(\Delta_{i} \circ \Delta_{j}\right) \Delta_{j}
\end{gathered}
$$

It is clear that the same change of basis of vanishing cycles obtained from operation $\alpha_{j}(i)$ is obtained by moving $t$ in clockwise direction as in Figure 3 .

We can now define a monodromy matrix $M$, which describes how the basis of cycles changes as $t$ is deformed in a complete circle. Because of the basis-change formula (14), the monodromy matrix depends on the intersection numbers. However, its eigenvalues can be computed explicitly in terms of the Ramond charges, and so it will yield an equation for the soliton numbers. To define $M$, we take the set of paths $\gamma_{i}$ to be straight lines (Figure 4). Because the soliton numbers have a simpler definition for an even number of fields let us assume $N$ is even. If $t$ is along the 


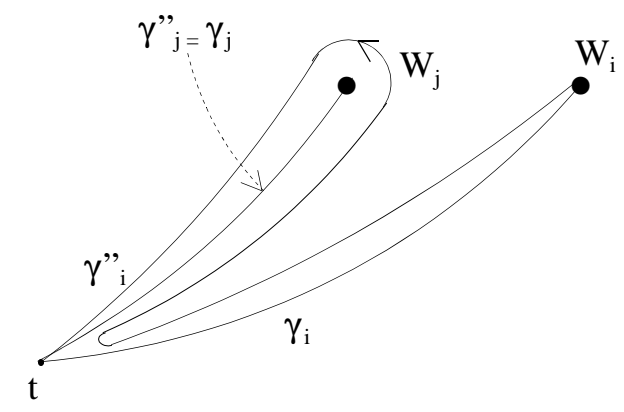

Figure 2: Change of paths operation $\omega_{j}(i)$

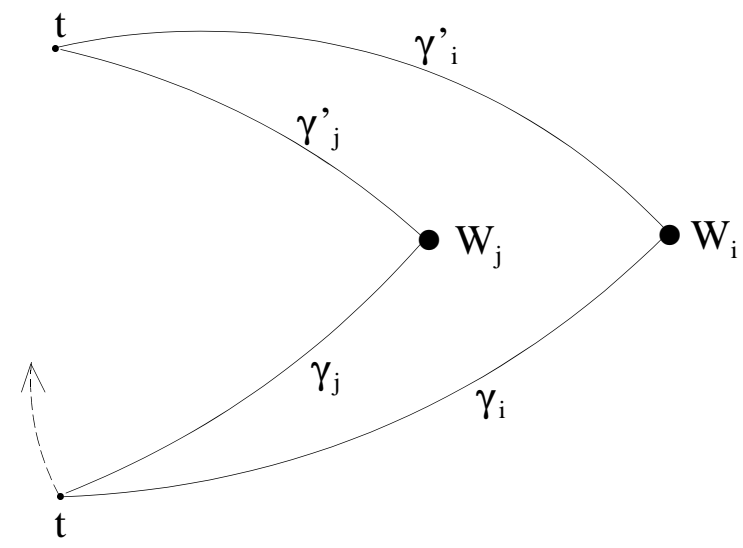

Figure 3: The change of basis is given by $\alpha_{j}(i)$

straight line connecting vacua $W_{i}$ and $W_{j}$, one has $\mu_{i j}=\Delta_{i} \circ \Delta_{j}$. Suppose instead $t$ is taken to be very far from the critical values. As $t$ is moved, $t$ will be collinear with pairs of vacua, and when this happens a change of basis occurs according to equation (14). If $t$ goes completely around a large circle in clockwise direction with $|t|$ fixed, the resulting change of basis is called a monodromy. Let $A_{i j}$ be the matrix whose only non-zero entry is the $(i, j)$ entry, which is equal to $\mu_{i j}=\Delta_{i} \circ \Delta_{j}$. The monodromy matrix $M$ is then an ordered product of change-of-basis matrices $M_{i j}=I+(-1)^{N(N+1) / 2} A_{i j}$. As $t$ goes halfway around the circle it will be collinear with all possible pairs of critical values; there are $p(p-1) / 2$ such pairs. We denote $S$ to be the ordered product of matrices $M_{i j}$ as $t$ goes half the way around the circle by:

$$
S=\prod_{\text {pairs }(i, j)} M_{i j}
$$

Since $M_{j i}=M_{i j}^{-t}$, as $t$ goes around the full circle the monodromy matrix is:

$$
M=S S^{-T} .
$$

The monodromy matrix contains information about the BPS spectrum, namely the soliton numbers between all pairs of vacua. The crucial point is that it can be computed in a completely different way [5]. It is known from singularity theory that the monodromy matrix is 


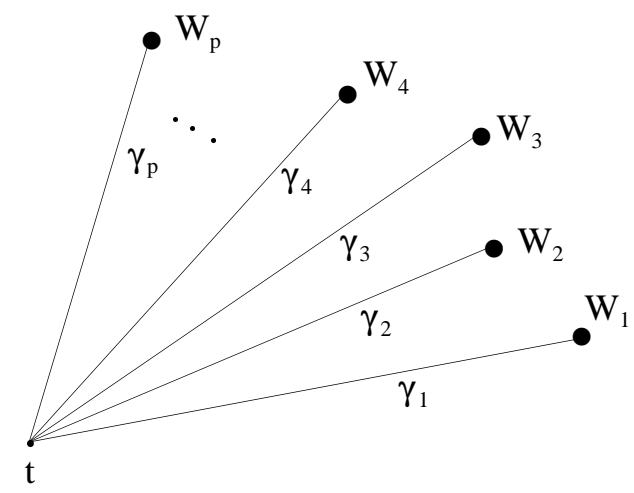

Figure 4: The set of paths $\gamma_{i}$ connecting $t$ to the critical values $W_{i}$ are straight lines

independent of finite deformations of the vacua. Moreover, one can compute the eigenvalues of the monodromy matrix directly in the superconformal limit $\lambda \rightarrow 0$, where all the $W_{i}$ are the same. They can be expressed in terms of $U(1)$ charges of the Ramond vacua $q_{m}^{R}$. For an even number of fields, one has [5]

$$
\text { eigenvals }(M)=\exp \left(2 \pi i q_{m}^{R}\right) .
$$

This is the key equation: the left-hand-side can be expressed solely in terms of the BPS soliton numbers, while the right hand side gives the spectrum of charges of chiral fields of the superconformal theory. This equation is quite profound: it directly relates a property of the infrared (the soliton spectrum) to that of the ultraviolet (the Ramond charges).

The assumption that the number of fields is even is not a restriction, because one can always add a non-interacting field $Y^{2}$ to the superpotential. Thus, for convenience in defining the soliton numbers in terms of intersection matrix of vanishing cycles, we consider the modified superpotential:

$$
W(X, Y)=\frac{X^{n+2}}{n+2}-\lambda \frac{X^{2}}{2}+Y^{2} .
$$

This modified superpotential and the original one have the same $X$-plane and $W$-plane vacua geometry. The addition of the field $Y^{2}$ only changes signs in the intersection matrix, and the actual soliton numbers are $\left|\mu_{a b}\right|$. Since for functions of an even number of variables the selfintersection matrix of vanishing cycles is zero, the physical requirement $\mu_{a a}=0$ follows directly. The exponentials $\exp \left(2 \pi i q_{m}^{R}\right)$ of the Ramond charges (12) for this theory are the zeroes of the polynomial $P(z)$

$$
P(z)=z^{n+1}-z^{n}+z^{n-1}-\ldots \pm 1 .
$$

with the last sign + for $n$ odd and - for $n$ even. Thus the relation (15) for the soliton numbers can be rewritten as:

$$
\operatorname{det}(M-z)=P(z) .
$$

To complete the computation we need to compute the monodromy matrix $M$. We do this in the next two sections. We treat separately the cases $n$ odd and $n$ even, because the geometry of the vacua in $W$-plane as well as the definitions of the soliton numbers differ slightly in the two cases. 


\section{The BPS spectrum for $n$ odd}

In this section we compute the soliton numbers (8) $\mu(i)$ in the case $n$ odd. When $n$ is odd, the critical values (vacua) (7) in $W$-plane are all distinct. The $\mathbb{Z}_{n}$-symmetry $X \rightarrow \exp (2 \pi i k / n) X$ in $X$-plane results in a $\mathbb{Z}_{n}$-symmetry in the $W$-plane which takes $W \rightarrow \exp (4 \pi i k / n) W$. The soliton numbers are defined by (13) in terms of the intersection matrix of vanishing cycles. The modified superpotential contains an even number of fields. Therefore the soliton numbers satisfy:

$$
\begin{aligned}
& \mu(-i)=-\mu(i), \quad i=1, \ldots,[n / 2] ; \\
& \mu_{0 i}=-\mu_{i 0}=\mu(0), \quad i=1, \ldots, n .
\end{aligned}
$$

To compute the monodromy matrix $M$, we take a non-vacuum point $t$ in $W$-plane far from the vacua just below the real axis with $\operatorname{Re}(t)<0$, and let it move in a clockwise direction around the critical values with $|t|=$ fixed (see figures 5 and 6 ). We consider all lines connecting the vacua which intersect, in order, the half circle obtained by rotating $t$ by $\pi$. There are $(n+1) / 2$ lines passing through each of the $n$ points (corners of the $n$-polygon) in $W$-plane, each line corresponding to one of $\mu(0), \mu(1), \ldots \ldots \mu\left(\left[\frac{n}{2}\right]\right)$.

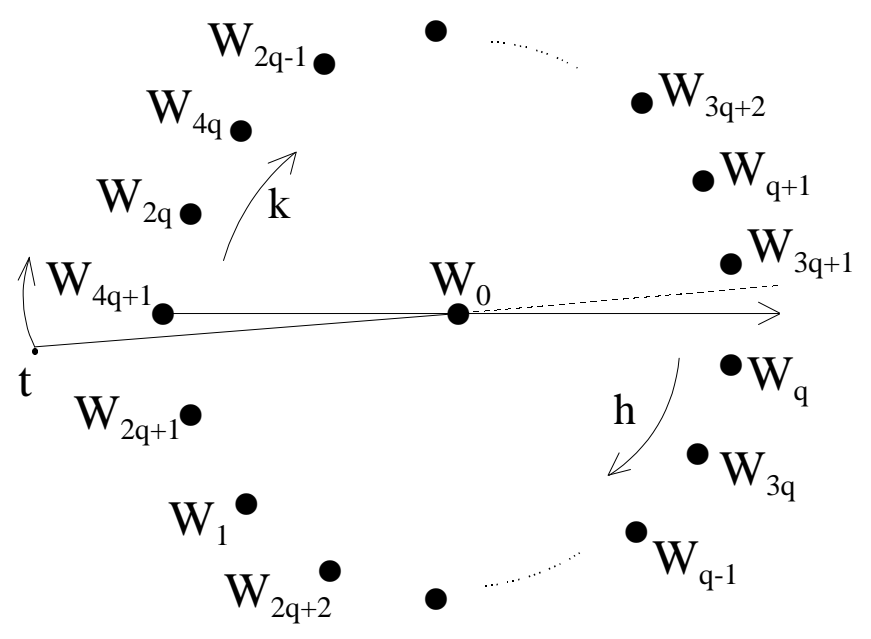

Figure 5: Vacua geometry in $W$-plane for $n=4 q+1$

The matrix $S$ consists of a product of $n(n+1) / 2$ change of basis matrices $M_{i j}=I-A_{i j}$. However, we can find a commuting set of $(n+1) / 2$ matrices $A_{i j}$ which contain each different soliton number $\mu(i)$ once. This suggests that one may be able to simplify the matrix $S$, and thus the monodromy matrix $M=S S^{-T}$, so that (17) can be solved for soliton numbers. Let us look at vacua in $W$-plane, and consider a line passing through any two vacua except the origin. There is a set of $(n-1) / 2$ such lines parallel to each other. If the point $t$ is far enough from the vacua, it crosses all these parallel lines sequentially as it is rotated. Moreover, the product of $A$ matrices corresponding to any two of these lines must vanish, since the parallel lines join different critical points. We add to this set the line through the origin and another critical point which appears (as $t$ is rotated) just before these parallel lines. The $A$ matrix for this line also gives zero when multiplied with the others in the set. This set of $(n+1) / 2$ lines therefore contributes to the matrix $S$ as $I-\mu^{<s>}$, where $\mu^{<s>}$ is the sum of all $A$ matrices associated 


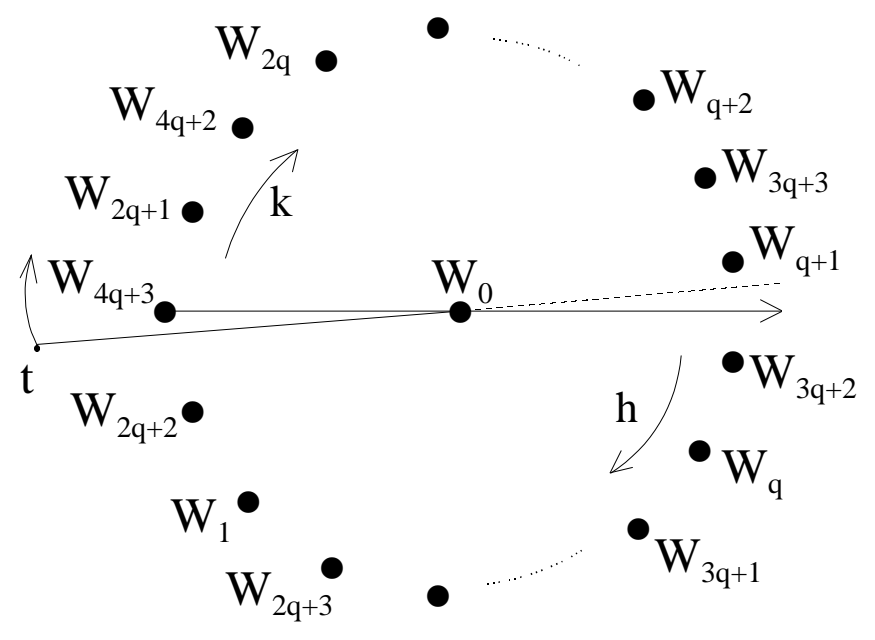

Figure 6: Vacua geometry in $W$-plane for $n=4 q+3$

with this set. There are $n$ such sets of lines, so the matrix $S$ can be written as:

$$
S=\left(I-\mu^{<1>}\right)\left(I-\mu^{<2>}\right) \ldots \ldots \ldots\left(I-\mu^{<n>}\right)
$$

To give an example, for $n=7$, the vacua form a pentagon, as displayed in figure 7 below. There are three $\mu^{\prime} s$ to be computed: $\mu(0), \mu(1), \mu(2)$. The matrix $S$ contains five sets each consisting of three matrices:

$$
S=\left(M_{05} M_{15} M_{42}\right)\left(M_{10} M_{35} M_{12}\right)\left(M_{02} M_{32} M_{14}\right)\left(M_{30} M_{52} M_{34}\right)\left(M_{04} M_{54} M_{31}\right)
$$

where $M_{i j}=I-A_{i j}$. The product of any two matrices $A$ in each set gives zero. Then:

$$
\begin{aligned}
S= & \left(I-A_{05}-A_{15}-A_{42}\right)\left(I-A_{10}-A_{35}-A_{12}\right)\left(I-A_{02}-A_{32}-A_{14}\right) \\
& \left(I-A_{30}-A_{52}-A_{34}\right)\left(I-A_{04}-A_{54}-A_{31}\right)
\end{aligned}
$$

Here

$$
\mu^{<1>}=A_{05}+A_{15}+A_{42}, \ldots, \mu^{<5>}=A_{04}+A_{54}+A_{31}
$$

To simplify $S$ further, we note that in each of these sets $\mu^{(s)}$, each different soliton number $\mu(i)(i=0,1 \ldots(n-1) / 2)$ appears exactly once. We can connect any matrix $A$ belonging to $\mu^{<s+1>}$ to one of $\mu^{<s>}$ in the same way, which then provides a connection between $\mu^{<s+1>}$ and $\mu^{<s>}$. Precisely, we will show that the monodromy matrix $M$ can be reduced to

$$
\begin{array}{lrl}
M & =\left[\left(I-\mu^{<1>}\right) R^{q}\left(I+\mu^{<1>}\right)^{T} R^{q}\right]^{n} & \text { for } n=4 q+1 \\
M & =\left[\left(I-\mu^{<1>}\right) R^{-q-1}\left(I+\mu^{<1>}\right)^{T} R^{-q-1}\right]^{n} & \text { for } n=4 q+3
\end{array}
$$

where $R$ is the $(n+1) \times(n+1)$ matrix:

$$
R=\left(\begin{array}{ccccccc}
1 & 0 & 0 & 0 & \ldots & 0 & 0 \\
0 & 0 & 0 & 0 & \ldots & 0 & 1 \\
0 & 1 & 0 & 0 & \ldots & 0 & 0 \\
0 & 0 & 1 & 0 & \ldots & 0 & 0 \\
. . & . . & . . & . . & . . & . . & . . \\
. . & . . & . . & . . & . . & . . & . . \\
0 & 0 & 0 & 0 & 0 & 1 & 0
\end{array}\right)
$$


$R$ satisfies: $R^{-n}=R^{n}=I$ and $R^{T}=R^{-1}$.

To derive (19) and (20), we use the notation $\langle r, s\rangle$ for the ray starting at $r$ and passing through $s$. There are $\frac{n-1}{2}$ points for which $\operatorname{Im}\left(W_{k}\right)>0$ and one point, $k=n$, for which $\operatorname{Im}\left(W_{n}\right)=0$. We list these points in order of decreasing phase (see Figure 5 and 6 ):

$$
\begin{aligned}
& k=\{4 q+1,2 q, 4 q, 2 q-1,4 q-1, \ldots ., q+1,3 q+1\} \quad \text { for } \quad n=4 q+1 \\
& k=\{4 q+3,2 q+1,4 q+2,2 q, 4 q+1,2 q-1, \ldots ., 3 q+3, q+1\} \quad \text { for } \quad n=4 q+3
\end{aligned}
$$

The list of points $W_{h}$ for which $\operatorname{Im}\left(W_{h}\right)<0$ in order of decreasing phase is:

$$
\begin{aligned}
& h=\{q, 3 q, q-1,3 q-1, \ldots ., 1,2 q+1\} \quad \text { for } \quad n=4 q+1 \\
& h=\{3 q+2, q, 3 q+1, q-1,3 q, \ldots . ., 1,2 q+2\} \quad \text { for } \quad n=4 q+3
\end{aligned}
$$

All $W_{k}$ are equally spaced, with an angle of $2 \pi / n$ between any two consecutive vacua. Let us look first at rays connecting vacuum $W_{0}$ with any other vacuum. As $t$ is rotated, consecutive rays alternate between those of the form $\langle 0, k\rangle$ and those of the form $\langle h, 0\rangle$. For $n=4 q+1$ the sequence of rays passing through $W_{0}$ has the following form, ordered as they appear in the computation of $S$ :

$$
\ldots\langle 0,4 q+1-j\rangle,\langle q-j, 0\rangle,\langle 0,2 q-j\rangle,\langle 3 q-j, 0\rangle \ldots
$$

The $A$ matrices corresponding to consecutive rays can be related by:

$$
\begin{aligned}
-A_{0,4 q+1-j}^{T} & =R^{-q} A_{q-j, 0} R^{q} \\
-A_{q-j, 0}^{T} & =R^{-q} A_{0,2 q-j} R^{q} \\
-A_{0,2 q-j}^{T} & =R^{-q} A_{3 q-j, 0} R^{q}
\end{aligned}
$$

and so on. For $n=4 q+3$ the sequence is:

$$
\ldots\langle 0,4 q+3-j\rangle,\langle 3 q+2-j, 0\rangle,\langle 0,2 q+1-j\rangle,\langle q-j, 0\rangle, \ldots
$$

and the corresponding relations are:

$$
\begin{aligned}
-A_{0,4 q+3-j}^{T} & =R^{q+1} A_{3 q+2-j, 0} R^{-q-1} \\
-A_{3 q+2-j, 0}^{T} & =R^{q+1} A_{0,2 q+1-j} R^{-q-1} \\
-A_{0,2 q+1-j}^{T} & =R^{q+1} A_{q-j, 0} R^{-q-1}
\end{aligned}
$$

The same connection exists between the matrices $A$ corresponding to lines which do not pass through vacuum $W_{0}$. We show this for $n=4 q+1$. Any point $k$ for which $\operatorname{Im}\left(W_{k}\right)>0$ is of the form $k=2 q-j$ or $k=4 q+1-j$, while for a point $W_{h}$ for which $\operatorname{Im}\left(W_{h}\right)<0, h$ can be of the form $h=q-l$ or $h=3 q-l$. Consider rays $\langle 2 q-j, q-l\rangle$ and $\langle 2 q-l, q-j\rangle$. These rays are parallel, as is easy to check by substituting the explicit expression for $W_{k}$ and $W_{h}$. Their $A$ matrices therefore both contribute to the same set $\mu^{<s>}$. The lines contributing to the next set $\mu^{<s+1>}$ then includes $\langle 3 q-l, 2 q-j\rangle$ and all lines parallel to it. Each $A$ matrix in $\mu^{(s)}$ is related to one in $\mu^{(s+1)}$ by a relation like

$$
-A_{q-j, 2 q-l}^{T}=R^{-q} A_{3 q-l, 2 q-j} R^{q} .
$$

Therefore the relation between a set $\mu^{<s+1>}$ and $\mu^{<s>}$ is:

$$
\left(I+\mu^{<s>}\right)^{T}=R^{-q}\left(I-\mu^{<s+1>}\right) R^{q} .
$$


Using this relation, the matrix $S$ becomes

$$
S=\left[\left(I-\mu^{<1>}\right) R^{q}\left(I+\mu^{<1>}\right)^{T} R^{q}\right]^{2 q}\left(I-\mu^{<1>}\right) R^{q},
$$

where $\mu^{<1>}$ is the sum of all $A$ matrices which appear first when computing $S$. These correspond to the parallel lines with the closest slope to $\pi$, along with the ray passing through the vacua $W_{0}$ and $W_{n}$. Because $\left(I-\mu^{<1>}\right)^{-T}=\left(I+\mu^{<1>}\right)^{T}$, the monodromy matrix reduces to the form given in equation (19). For $n=4 q+3$ the manipulations are similar and the monodromy matrix $M$ is given in equation (20).

We can finally solve for the soliton numbers in (17) by using the explicit expressions for the monodromy matrix coming from (19) or (20). The Ramond charges (12) are symmetrically distributed between $-3 c / 2$ and $3 c / 2$ (where $c=3 n /(n+2)$ is the central charge of the conformal theory). Thus the $\lambda_{m}=\exp \left(2 \pi i q_{m}^{R}\right)$ and hence the eigenvalues of $M$ are all phases and distinct. This means that the eigenvalues $\beta_{m}$ of $M^{1 / n} \equiv\left(I-\mu^{<1>}\right) R^{q}\left(I+\mu^{<1>}\right)^{T} R^{q}$ are phases and distinct as well. This means that $M^{1 / n}$ is also a monodromy, that is, it does not depend on perturbations. In fact, both the group of automorphisms Aut $H_{1}\left(V_{t}\right)$ of the homology group $H_{1}\left(V_{t}\right)$ and the fundamental group $\pi_{1}(t)$ factorize under $\mathbb{Z}_{n}$ into $n$ identical subgroups. Then the image of the homomorphism of such a subgroup of $\pi_{1}(t)$ in a subgroup of $A u t H_{1}\left(V_{t}\right)$ defines a monodromy. When all critical values are identical the eigenvalues of $\left(I-\mu^{<1>}\right) R^{q}\left(I+\mu^{<1>}\right)^{T} R^{q}$ are again given as exponentials of the Ramond charges. This is consistent with the fact that the eigenvalues of $M$ are the same exponentials of Ramond charges, because the set $\left(\beta_{m}\right)^{n}$ is the same as the set of $\lambda_{m}$. Therefore the eigenvalues of $M$ and $M^{1 / n}$ are identical, and we can substitute the latter for the former when solving (17). We can $\operatorname{transform~} \operatorname{det}\left(z-M^{1 / n}\right)$ by using the facts that $\operatorname{det}\left(I-\mu^{<1>}\right)=\operatorname{det}(R)=1$ :

$$
\begin{aligned}
\operatorname{det}\left[z-\left(I-\mu^{<1>}\right) R^{q}\left(I+\mu^{<1>}\right)^{T} R^{q}\right] & =\operatorname{det}\left[z R^{-q}\left(I-\mu^{<1>}\right)^{T}-\left(I-\mu^{<1>}\right) R^{q}\right] \\
& =\operatorname{det}\left\{R^{q}\left[z R^{-q}\left(I-\mu^{<1>}\right)^{T}-\left(I-\mu^{<1>}\right) R^{q}\right] R^{-q}\right\} \\
& =\operatorname{det}\left[z\left[R^{q}\left(I-\mu^{<1>}\right)\right]^{T}-R^{q}\left(I-\mu^{<1>}\right)\right] .
\end{aligned}
$$

After doing the same transformation for $n=4 q+3$, the equations to be solved for soliton numbers become:

$$
\begin{aligned}
\operatorname{det}\left[z\left[R^{q}\left(I-\mu^{<1>}\right)\right]^{T}-R^{q}\left(I-\mu^{<1>}\right)\right] & =P(z), \quad \text { for } \quad n=4 q+1 \\
\operatorname{det}\left[z\left[R^{-q-1}\left(I-\mu^{<1>}\right)\right]^{T}-R^{-q-1}\left(I-\mu^{<1>}\right)\right] & =P(z), \quad \text { for } \quad n=4 q+3 .
\end{aligned}
$$

We can now use the explicit expressions for $\mu^{<1>}$. For $n=4 q+1$, it is

$$
\begin{aligned}
\left(\mu^{<1>}\right)_{i j}= & \delta_{i, 0} \delta_{j, 4 q+1} \mu(0)-\delta_{i, q} \delta_{j, 4 q+1} \mu(q)-\delta_{i, 3 q+1} \delta_{j, 2 q} \mu(q+1)-\delta_{i, q+1} \delta_{j, 4 q} \mu(q+2) \\
& -\delta_{i, 3 q+2} \delta_{j, 2 q-1} \mu(q+3)-\delta_{i, q+2} \delta_{j, 4 q-1} \mu(q+4)-\ldots-\delta_{i, 3 q} \delta_{j, 2 q+1} \mu(q-1) \\
& -\delta_{i, q-1} \delta_{j, 1} \mu(q-2)-\delta_{i, 3 q-1} \delta_{j, 2 q+2} \mu(q-3)-\delta_{i, q-2} \delta_{j, 2} \mu(q-4)-\ldots
\end{aligned}
$$

where we have used $\mu(-i)=-\mu(i)$ and $\mu(i-n)=\mu(i)$. The matrix is of the form

$$
I-\mu^{<1>}=\left(\begin{array}{cccccc}
1 & & & & & -\mu(0) \\
& B & & & & A \\
& & I_{q} & & C & \\
& & & D & & \\
& & E & & I_{q} & \\
& & & & & 1
\end{array}\right),
$$


where omitted entries are zero, $I_{q}$ is the $q \times q$ identity, $A$ is a column of length $q$ and $B, C, D, E$ are $q \times q$ matrices. For example for $q=5, n=21$ they are:

$$
\begin{gathered}
A=\left(\begin{array}{l}
0 \\
0 \\
0 \\
0 \\
\mu(5)
\end{array}\right), B=\left(\begin{array}{lllll}
1 & 0 & 0 & 0 & 0 \\
0 & 1 & 0 & 0 & 0 \\
0 & \mu(1) & 1 & 0 & 0 \\
\mu(3) & 0 & 0 & 1 & 0 \\
0 & 0 & 0 & 0 & 1
\end{array}\right), C=\left(\begin{array}{lllll}
0 & 0 & 0 & 0 & \mu(7) \\
0 & 0 & 0 & \mu(9) & 0 \\
0 & 0 & 0 & 0 & 0 \\
0 & 0 & 0 & 0 & 0 \\
0 & 0 & 0 & 0 & 0
\end{array}\right) \\
D=\left(\begin{array}{lllll}
1 & 0 & 0 & 0 & 0 \\
0 & 1 & 0 & 0 & 0 \\
0 & 0 & 1 & 0 & 0 \\
0 & \mu(2) & 0 & 1 & 0 \\
\mu(4) & 0 & 0 & 0 & 1
\end{array}\right), E=\left(\begin{array}{lllll}
0 & 0 & 0 & 0 & \mu(6) \\
0 & 0 & 0 & \mu(8) & 0 \\
0 & 0 & \mu(10) & 0 & 0 \\
0 & 0 & 0 & 0 & 0 \\
0 & 0 & 0 & 0 & 0
\end{array}\right)
\end{gathered}
$$

Multiplication to the left by $R^{q}$ shifts the rows, except for row 0 . The computation of the determinant gives:

$$
\begin{aligned}
P(z)= & z^{n+1}-z^{n}(1-\mu(2 q))-z(1-\mu(2 q))+1-z^{\frac{n+1}{2}}\left(-\mu^{2}(0)-2 \mu(q)\right)+ \\
& +\sum_{k=1}^{2 q} z^{n-2 k+1}(-\mu(k)-\mu(2 q-k+1))-\sum_{k=1, k \neq q}^{2 q-1}(-\mu(k)-\mu(2 q-k)) .
\end{aligned}
$$

By comparing the above polynomial with the form of $P(z)$ given by (17), one finds

$$
\begin{aligned}
\mu(0) & = \pm 1 \\
\mu(1) & =\mu(2)=\mu(3)=\ldots=\mu(q)=-1 \\
\mu(q+1) & =\mu(q+2)=\ldots=\mu(2 q+1)=0
\end{aligned}
$$

The computation for $n=4 q+3$ is similar. One has

$$
\begin{aligned}
\left(\mu^{<1>}\right)_{i j}= & \delta_{i, 0} \delta_{j, 4 q+3} \mu(0)+\delta_{i, q+1} \delta_{j, 2 q+1} \mu(q)+\delta_{i, 3 q+2} \delta_{j, 4 q+3} \mu(q+1)+\delta_{i, 3 q+3} \delta_{j, 4 q+2} \mu(q-1)+ \\
& +\delta_{i, q+2} \delta_{j, 2 q} \mu(q-2)+\delta_{i, 3 q+4} \delta_{j, 4 q+1} \mu(q-3)+\delta_{i, q+3} \delta_{j, 2 q-1} \mu(q-4)+\ldots+ \\
& +\delta_{i, q} \delta_{j, 2 q+2} \mu(q+2)+\delta_{i, 3 q+1} \delta_{j, 1} \mu(q+3)+\delta_{i, q-1} \delta_{j, 2 q+3} \mu(q+4)+\ldots
\end{aligned}
$$

Then $I-\mu^{<1>}$ has the form:

$$
I-\mu^{<1>}=\left(\begin{array}{cccc}
I_{q+1} & & A & B \\
& C & & \\
D & & I_{q+1} & F \\
& & & E
\end{array}\right),
$$

where $A, B, C, D, E, F$ are $(q+1) \times(q+1)$ matrices. Taking the determinant yields

$$
\begin{aligned}
P(z)= & z^{n+1}-z^{n}(1-\mu(2 q+1))-z(1-\mu(2 q+1))+1+z^{\frac{n+1}{2}}\left(\mu^{2}(0)-2 \mu(q+1)\right)+ \\
& +\sum_{k=1, k \neq q+1}^{2 q+1} z^{n-2 k+1}(-\mu(k)-\mu(2 q-k+2))-\sum_{k=1}^{2 q} z^{n-2 k}(-\mu(k)-\mu(2 q-k+1)) .
\end{aligned}
$$

This yields soliton numbers identical to those for $n=4 q+1$, as given in (21). 

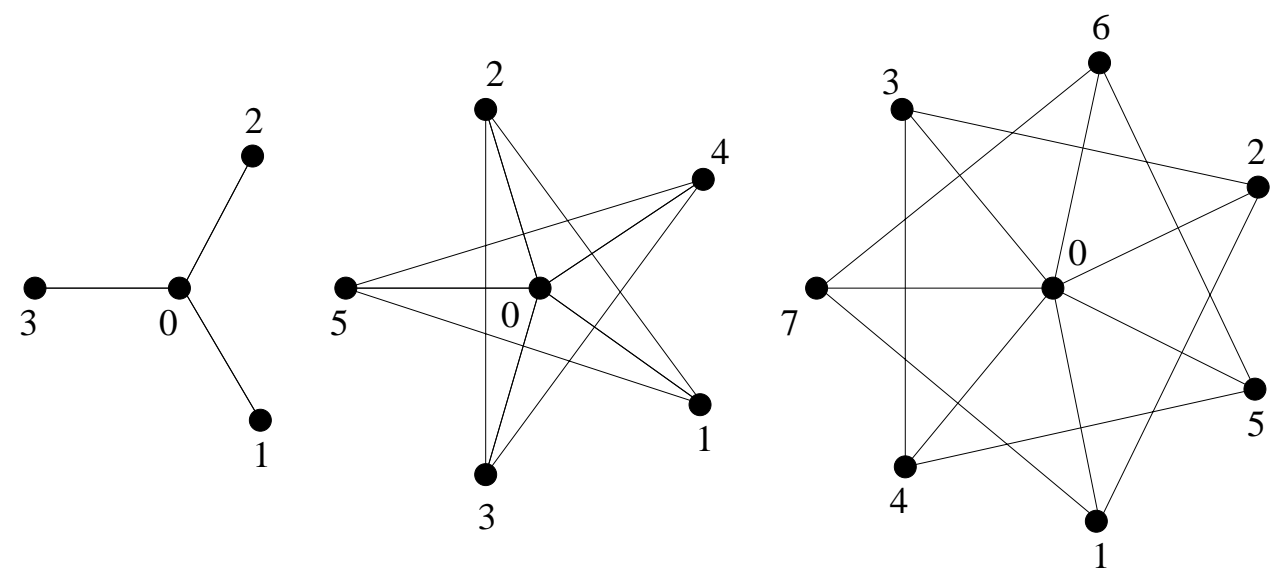

Figure 7: Solitons in $W$-plane for $n=3,5,7$. Straight lines represent BPS states.

We have finally arrived at our answer for $n$ odd. For the first three cases, the soliton numbers are displayed graphically in figure 7; we draw a solid line to denote each doublet. The pattern is fairly obvious. There is always a single doublet of fractional charges $(1 / 2,-1 / 2)$ connecting the origin to each of the other vacua. For solitons not involving the origin, there is a single doublet connecting each vacuum to its $2[n / 4]$ nearest neighbors in the $X$ plane. Nearest neighbors in the $X$ plane are not necessarily nearest neighbors in the $W$ plane, as is evident from the figure. Note also that the soliton doublets not involving the vacuum 0 have either fractional charge $(0,1)$ or $(0,-1)$. $C P$ invariance means that if the doublet $(a b)$ has charge $(0,1)$, then the doublet $(b a)$ must have charges $(0,-1)$. The $\mathbb{Z}_{n}$ symmetry means that all clockwise solitons (say) are the former, all counterclockwise solitons are the latter.

\section{The BPS spectrum for $n$ even}

Finding the BPS spectrum for $n$ even requires more results from Picard-Lefschetz theory, because the critical values in $W$-plane are not all distinct. Two critical points diametrically opposed in the $X$ plane map into the same critical value in $W$ plane. Therefore two vanishing cycles $\Delta_{i}, \Delta_{i+n / 2}$ correspond to each $W_{i}$ with $i=1 \ldots n / 2$, while one vanishing cycle $\Delta_{0}$ corresponds to the critical value at the origin in $W$-plane. The intersection matrix of two vanishing cycles corresponding to two critical points that map into the same critical value is zero, so $\Delta_{i} \circ \Delta_{i+n / 2}=$ $\mu\left(\left[\frac{n}{2}\right]\right)=0$. This can also be seen directly from equation (5), because a BPS soliton satisfying $\Delta W=0$ must in fact be a constant value of $X$.

For $n$ even, the homology group splits naturally into two subspaces. As before, the homology group $H_{1}\left(V_{t}\right)$ of the non-singular level manifold $V_{t} \equiv W^{-1}(t)$ consists of $n+1$ vanishing cycles $\left\{\Delta_{0}, \Delta_{1}, \ldots, \Delta_{n}\right\}$, corresponding to the $n+1$ critical points. However, here the superpotential $W$ is invariant under the involution $\sigma$ on $\mathbb{C}^{2}: \sigma(X, Y)=(-X, Y)$. The involution $\sigma$ acts on the vanishing cycles as 18]:

$$
\sigma_{*} \Delta_{i}=\Delta_{i+n / 2}, \quad \sigma_{*} \Delta_{0}=-\Delta_{0}
$$

This shows that the cycles $\Delta_{i}$ and $\Delta_{i+n / 2}$ have different orientations. Since $\mathbb{Z}_{2}$ has two real irreducible representations, the homology group $H_{1}\left(V_{t}\right)$ decomposes into a direct sum $H^{+} \oplus H^{-}$, where $H^{+}$contains the vanishing cycles invariant under the involution $\sigma$, and $H^{-}$the anti- 
invariant ones. A basis in $H^{+}$is given by 'long-cycles' $\widetilde{\Delta}_{i}$ with $i=1 \ldots n / 2$ :

$$
\widetilde{\Delta}_{i} \equiv \Delta_{i}+\Delta_{i+n / 2}
$$

while a basis in $H^{-}$is given by 'short-cycle' $\Delta_{0}$, and long-cycles $\widehat{\Delta}_{i}$ with $i=1 \ldots n / 2$ :

$$
\widehat{\Delta}_{i} \equiv \Delta_{i}-\Delta_{i+n / 2}
$$

Under the $\mathbb{Z}_{2}$, these cycles obey $\sigma_{*} \widetilde{\Delta}_{i}=\widetilde{\Delta}_{i}$ and $\sigma_{*} \widehat{\Delta}_{i}=-\widehat{\Delta}_{i}$, respectively. Intersections between cycles corresponding to different subgroups of the homology group are zero:

$$
\begin{aligned}
& \Delta_{0} \circ \widetilde{\Delta}_{i}=0 \\
& \widehat{\Delta}_{i} \circ \widetilde{\Delta}_{j}=0
\end{aligned}
$$

The change of basis formula in Picard-Lefschetz theory depends on whether the cycle being crossed is long or short [18, 24]. When the path $\gamma_{a}$ to a vacuum $W_{a}$ crosses a long cycle $\gamma_{b}$, $b \neq 0$, the change of basis $\alpha_{b}(a)$ is

$$
\Delta_{a} \rightarrow \Delta_{a}^{\prime}=\Delta_{a}-\left(\Delta_{a} \circ \Delta_{b}\right) \Delta_{b} / 2
$$

whereas when it crosses a short cycle, the change of basis $\alpha_{0}(a)$ is

$$
\Delta_{a} \rightarrow \Delta_{a}^{\prime}=\Delta_{a}-\left(\Delta_{a} \circ \Delta_{0}\right) \Delta_{0}
$$

One can associate the invariant and anti-invariant cycles to critical points of different functions. Let us consider the transformation: $\widehat{X}=X^{2}, \widehat{Y}=Y$. Then $\widehat{X}, \widehat{Y}$ are coordinates on the manifold $\mathbb{C}^{2} / \mathbb{Z}_{2} \simeq \mathbb{C}^{2}$ with boundary at $\widehat{X}=0$. The two-dimensional hyperplane $\mathbb{C}^{2}$ with coordinates $X, Y$ can be viewed as the double covering of $\mathbb{C}^{2} / \mathbb{Z}_{2} \simeq \mathbb{C}^{2}$ with a branch on the boundary $\widehat{X}=0$. This transformation induces the function on $\mathbb{C}^{2} / \mathbb{Z}_{2}$ :

$$
\widehat{W}\left(\widehat{X}^{2}, \widehat{Y}\right) \equiv W(X, Y) \text {, with } \widehat{W}(\widehat{X}, \widehat{Y}) \equiv \frac{\widehat{X}^{(n+2) / 2}}{n+2}-\lambda \frac{\widehat{X}}{2}+\widehat{Y}^{2}
$$

It can be shown 18, 25] that the number of critical points (the Milnor multiplicity) $\nu$ of functions $W$ and $\widehat{W}$ satisfy:

$$
\nu(W)=2 \nu(\widehat{W})+\nu(\widehat{W} \mid\{\widehat{X}=0\})
$$

and:

$$
\begin{gathered}
\operatorname{dim} H^{+}=\nu(\widehat{W})=n / 2, \\
\operatorname{dim} H^{-}=\nu(\widehat{W})+\nu(\widehat{W} \mid\{\widehat{X}=0\})=n / 2+1
\end{gathered}
$$

It is known that the analysis of singularities of functions $(W)$ invariant under $\mathbb{Z}_{2}$ is equivalent to the analysis of singularities of function $(\widehat{W})$ on manifolds with boundary [17, 18]. The analysis of singularities of boundary singularity, that is of the function $\widehat{W}+\left.\widehat{W}\right|_{\widehat{X}=0}$, can be carried out with the anti-invariant vanishing cycles, while the invariant cycles can be used for the singularities of $\widehat{W}$. The characteristic polynomial $P(z)$ factorizes into two polynomials $P^{+}(z)$ and $P^{-}(z)$ corresponding to the invariant and anti-invariant part respectively:

$$
P(z)=P^{+}(z) P^{-}(z)
$$


Intersection numbers of cycles in different subgroups vanish, so the monodromy matrix $M$ can be computed independently on each subspace of the homology group of vanishing cycles. It has the form:

$$
M=\left(\begin{array}{ll}
M^{+} & 0 \\
0 & M^{-}
\end{array}\right),
$$

where $M^{+}$is an $n / 2 \times n / 2$ matrix and $M^{-}$an $(n / 2+1) \times(n / 2+1)$ matrix. Then, equation (17) becomes:

$$
\begin{aligned}
\operatorname{det}\left(M^{-}-z\right) & =P^{-}(z) \\
\operatorname{det}\left(M^{+}-z\right) & =P^{+}(z)
\end{aligned}
$$

where the polynomials are

$$
P^{+}(z)=z^{n / 2}-z^{n / 2-1}+z^{n / 2-2}-\ldots \mp 1, \quad P^{-}(z)=z^{n / 2+1} \pm 1
$$

with the last signs $(-,+)$ for $n=4 q+2$ and $(+,-)$ for $n=4 q$. To write the monodromy matrices $M^{+}, M^{-}$in terms of the soliton numbers, we need to look at vacua in $W$-plane. There is a difference in the geometry of vacua in $W$-plane in the cases $n=4 q+2$ and $n=4 q$. When $n=4 q$ there are three different vacua which are collinear, situation that does not appear in the case $n=4 q+2$. Due to this difference we treat the two cases separately.

\subsection{BPS spectrum for $n=4 q+2$}

The homology group splits in two, and the intersection numbers for cycles in different subspaces is zero. Finding the soliton numbers for $n=4 q+2$ splits into two problems, one of which in fact has been solved before.

One additional complication is that the soliton numbers are of course invariant under the involution $\sigma$, but as detailed above, the intersection numbers are not (because the vanishing cycles are not (22)). This requires a slight modification of the relation between the two. The trajectory between vacua $X_{0}$ and $X_{i}$ with $1 \leq i \leq n / 2$, and that between $X_{0}$ and $X_{i+n / 2}$ map into the same straight line in $W$-plane. Since $\Delta_{i}$ and $\Delta_{i+n / 2}$ have different orientations, $\Delta_{0} \circ \Delta_{i}$ and $\Delta_{0} \circ \Delta_{i+n / 2}$ count the intersections points with different signs. Thus

$$
\mu(0) \equiv \mu_{0 i}=\mu_{0, i+n / 2}=\Delta_{0} \circ \Delta_{i}=-\Delta_{0} \circ \Delta_{i+n / 2} .
$$

The situation is similar for the other solitons. When defining the soliton number between two critical points $W_{i}$ and $W_{k}$, with $1 \leq i \leq n / 2$, and $n / 2<k \leq n$, one must again include a minus sign. This yields

$$
\begin{aligned}
& \mu_{i j}=\Delta_{i} \circ \Delta_{j}, \quad \text { for } \quad 1 \leq i, j \leq n / 2 \\
& \mu_{i k}=-\Delta_{i} \circ \Delta_{k}, \quad \text { for } \quad 1 \leq i \leq n / 2, \quad n / 2<k \leq n, \\
& \mu_{k l}=\Delta_{k} \circ \Delta_{l}, \quad \text { for } \quad n / 2<k, l \leq n
\end{aligned}
$$

The model has a $\mathbb{Z}_{n}$ symmetry as before, although in the $W$ plane this is only $\mathbb{Z}_{n / 2}$. These symmetries relate the soliton numbers $\mu_{i j}$, as before. On the invariant space,

$$
\begin{aligned}
\Delta_{0} \circ \widetilde{\Delta}_{i} & =0 \\
\widetilde{\Delta}_{i} \circ \widetilde{\Delta}_{j} & =2 \mu(j-i)-2 \mu(j-i-n / 2)
\end{aligned}
$$


which yields

$$
\begin{aligned}
\widetilde{\Delta}_{i} \circ \widetilde{\Delta}_{i+p} & =2 \mu(p)+2 \mu(2 q-(p-1)) \equiv 2 \widetilde{\mu}(p) \\
\widetilde{\Delta}_{2 q+1} \circ \widetilde{\Delta}_{1} & =-2 \mu(1)-2 \mu(2 q)
\end{aligned}
$$

for $p=1 \ldots q$ such that $1 \leq i+p \leq 2 q+1$. Therefore in the invariant part there are $q$ 'soliton numbers' $\widetilde{\mu}, \widetilde{\mu}_{i j} \equiv \frac{1}{2} \widetilde{\Delta}_{i} \circ \widetilde{\Delta}_{j}$ which satisfy:

$$
\begin{aligned}
\widetilde{\mu}_{i j} & =\widetilde{\mu}(j-i) \\
\widetilde{\mu}(k) & =-\widetilde{\mu}\left(k-\frac{n}{2}\right)
\end{aligned}
$$

for $k=1 \ldots q$. On the anti-invariant space the intersection numbers are:

$$
\begin{aligned}
& \Delta_{0} \circ \widehat{\Delta}_{i}=2 \mu(0) \\
& \widehat{\Delta}_{i} \circ \widehat{\Delta}_{j}=2 \mu(j-i)+2 \mu(j-i-n / 2)
\end{aligned}
$$

which give:

$$
\begin{aligned}
\widehat{\Delta}_{i} \circ \widehat{\Delta}_{i+p} & =2 \mu(p)-2 \mu(2 q-(p-1)) \equiv 2 \widehat{\mu}(p) \\
\widehat{\Delta}_{2 q+1} \circ \widehat{\Delta}_{1} & =2 \mu(1)-2 \mu(2 q)
\end{aligned}
$$

for all $p=1 \ldots q$ such that $1 \leq i+p \leq 2 q+1$. Therefore on the anti-invariant part there are $q+1$ 'soliton numbers': $\mu(0)$ and $\widehat{\mu}$, with $\widehat{\mu}_{i j} \equiv \frac{1}{2} \widehat{\Delta}_{i} \circ \widehat{\Delta}_{j}$. These satisfy:

$$
\begin{aligned}
\widehat{\mu}_{i j} & =\widehat{\mu}(j-i) \\
\widehat{\mu}(k) & =\widehat{\mu}\left(k-\frac{n}{2}\right),
\end{aligned}
$$

for $k=1 \ldots q$.

The above properties of the $\tilde{\mu}^{\prime} s$ are exactly the same as those satisfied by the real soliton numbers corresponding to the superpotential $\widehat{W}(\widehat{X}, \widehat{Y})=\frac{\widehat{X}^{(n+2) / 2}}{n+2}-\lambda \frac{\widehat{X}}{2}+\widehat{Y}^{2}$. These soliton numbers are known from the exact solution of this model in [11], and have been computed in [5] using the Picard-Lefschetz techniques. In our conventions, the result is that all $\widetilde{\mu}=-1$. Thus the invariant part has been solved and, in terms of the original soliton numbers, we have:

$$
\mu(p)+\mu(2 q-(p-1))=-1, p=1, \ldots, q
$$

To compute the soliton numbers $\widehat{\mu}$ in the anti-invariant sector, we compute the monodromy matrix $M^{-}$and use equation (23). The vacua geometry in $W$-plane is represented in Figure 8. We construct $M^{-}$in the same way as we did for $n$ odd, but because of the factors of 2 in various places, this will be slightly more complicated. Like before, the result can be simplified by relating basis matrices appearing sequentially. Here the sequence of critical values in $W$-plane is

$$
\begin{aligned}
k & =\{2 q+1,2 q, 2 q-1,2 q-2, \ldots . ., q+3, q+2, q+1\} \\
h & =\{q, q-1, q-2, q-3, \ldots ., 3,2,1\}
\end{aligned}
$$

where $\operatorname{Im}\left(W_{k}\right) \geq 0$, while $\operatorname{Im}\left(W_{h}\right) \leq 0$. The matrix $A_{0 k}$ (which arises when $t, W_{0}$ and $W_{k}$ are collinear) has $\mu(0)$ for its only non-zero entry $(0, k)$. However the change-of-basis matrix arising when $t, W_{0}$ and $W_{h}$ are collinear contains the entry $2 \mu(0)$. Let us therefore denote by $\widehat{A}_{h 0}$ the 


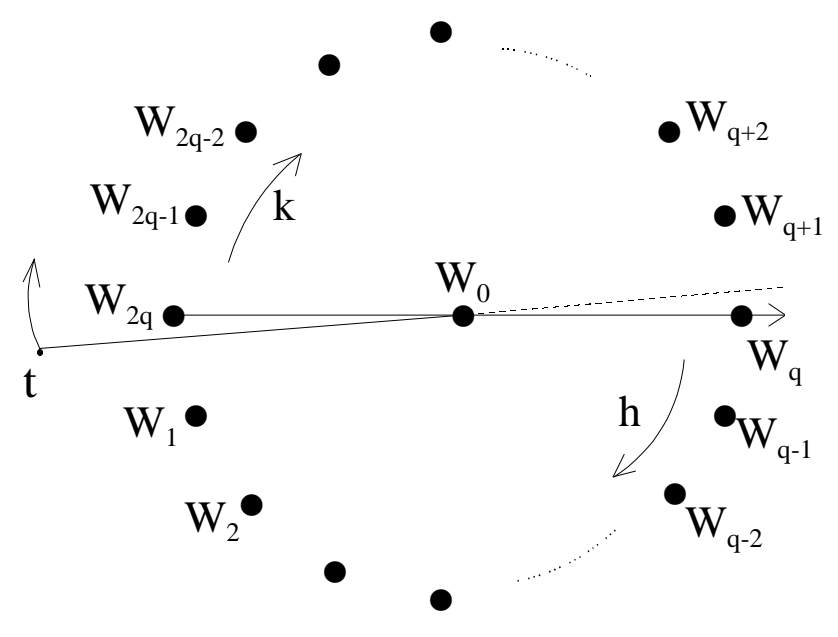

Figure 8: Vacua geometry in $W$-plane for $n=4 q+2$

matrix having $2 \mu(0)$ at the entry $(h, 0)$ and zero in rest. We can connect such $A$ and $\widehat{A}$ matrices, ordered as they appear when computing $M^{-}$, in the following way $(0 \leq i \leq q-1)$ :

$$
\begin{aligned}
R^{-q} \widehat{A}_{q-i, 0} R^{q} & =-2 A_{0,2 q+1-i}^{T} \\
R^{-q} A_{0,2 q-i} R^{q} & =-\frac{1}{2} \widehat{A}_{q-i, 0}^{T}
\end{aligned}
$$

Let us consider now the lines connecting two vacua except the origin, so that this involves only long cycles. The change of basis for when $t, W_{i}$ and $W_{j}$ are collinear is $M_{i j}=I-A_{i j}$, where $A_{i j}$ has $(i, j)$ the only non-zero entry equal to $\widehat{\mu}_{i j}$. The change of such basis matrices for such lines can be related again using matrices $R^{q}$ and $R^{-q}$. This yields the monodromy matrix:

$$
M^{-}=\left[\left(I-\mu_{1}^{\langle 1\rangle}\right) R^{q}\left(I+\mu_{2}^{\langle 1\rangle}\right)^{T} R^{q}\right]^{n / 2},
$$

where:

$$
\begin{aligned}
& \left(\mu_{1}^{\langle 1\rangle}\right)_{i j}=\delta_{i, 0} \delta_{j, 2 q+1} \mu(0)+\left(\mu^{\langle 1\rangle}\right)_{i j} \\
& \left(\mu_{2}^{\langle 1\rangle}\right)_{i j}=2 \delta_{i, 0} \delta_{j, 2 q+1} \mu(0)+\left(\mu^{\langle 1\rangle}\right)_{i j}
\end{aligned}
$$

As before, $\mu^{<1>}$ is the sum of all matrices $A_{i j}$ corresponding to the parallel lines which appear first (after the ray connecting $W_{0}$ and $W_{2 q+1}$ ). Explicitly, it is

$$
\begin{aligned}
\left(\mu^{\langle 1\rangle}\right)_{i j}= & -\delta_{i, q} \delta_{j, 2 q+1} \widehat{\mu}(q)+\delta_{i, q+1} \delta_{j, 2 q} \widehat{\mu}(q-1)+\delta_{i, q+2} \delta_{j, 2 q-1} \widehat{\mu}(q-3)+ \\
& +\delta_{i, q+3} \delta_{j, 2 q-2} \widehat{\mu}(q-5)+\ldots \ldots-\delta_{i, q-1} \delta_{j, 1} \widehat{\mu}(q-2)-\delta_{i, q-2} \delta_{j, 2} \widehat{\mu}(q-4)- \\
& -\delta_{i, q-3} \delta_{j, 3} \widehat{\mu}(q-6)-\delta_{i, q-4} \delta_{j, 4} \widehat{\mu}(q-8)-\ldots
\end{aligned}
$$

As for $n$ odd, solving equation (23) for $\widehat{\mu}^{\prime} s$ reduces to solving:

$$
\operatorname{det}\left[\left(I-\mu_{1}^{\langle 1\rangle}\right) R^{q}\left(I+\mu_{2}^{\langle 1\rangle}\right)^{T} R^{q}-z\right]=P^{-}(z) .
$$


Computing the determinant yields

$$
\begin{aligned}
P^{-}(z)= & z^{2 q+2}+1-z^{2 q+1}(1+\widehat{\mu}(1))-z(1+\widehat{\mu}(1))+z^{q+1}\left(2 \mu^{2}(0)+2 \widehat{\mu}(q)\right)+ \\
& +\sum_{k=0}^{q-2}\left[(-1)^{k} z^{2 q-k}(\widehat{\mu}(k+1)-\widehat{\mu}(k+2))+(-1)^{q-k} z^{q-k}(\widehat{\mu}(q-k-1)-\widehat{\mu}(q-k))\right] .
\end{aligned}
$$

Thus plugging in the polynomial from (23)

$$
\mu(0)= \pm 1, \widehat{\mu}(i)=-1, i=1, \ldots, q
$$

which in terms of the original $\mu^{\prime} s$ give:

$$
\mu(p)-\mu(2 q-(p-1))=-1, p=1, \ldots, q .
$$

Reexpressing this in terms of the original soliton numbers yields (after taking absolute values)

$$
\begin{gathered}
\mu(0)=1 \\
\mu(1)=\mu(2)=\mu(3)=\ldots=\mu\left(\left[\frac{n}{4}\right]\right)=1 \\
\mu\left(\left[\frac{n}{4}\right]+1\right)=\mu\left(\left[\frac{n}{4}\right]+2\right)=\ldots=\mu\left(\left[\frac{n}{2}\right]\right)=0
\end{gathered}
$$

So this is essentially the same as for $n$ odd: there is a single BPS doublet joining the origin to each vacuum, and a single BPS doublet joining a vacuum to its $2 q$ neighbors in the $X$-plane.

\section{$5.2 \quad$ BPS spectrum for $n=4 q$}

The case $n=4 q$ is the most complicated. This is because the vacua $W_{0}, W_{i}$ and $W_{i+q}$ are collinear for any $i=1 \ldots q$. Collinear vacua result in contributions to the index $C$ from multiparticle states that get mixed in with those from one-particle states. Specifically, $\mu_{a b}$ is defined as the coefficient of $C_{a b}$ in the $\beta \rightarrow \infty$ limit, as shown in (11). Since BPS solitons are constrained to have mass $m_{a b}=\left|\Delta_{a b}\right|$, the one-particle states indeed generically provide the only contributions to $C_{a b}$ at $\beta$ large. The one situation where the multi-particle contributions can be just as large is if there is some vacuum $c$ collinear with and in between $a$ and $b$. The central terms are additive in general: $\Delta_{a b}=\Delta_{a c}+\Delta_{c b}$ (this is obvious in a Landau-Ginzburg theory, where $\left.\Delta_{a b}=W(b)-W(a)\right)$. The special property of collinear vacua is that $\left|\Delta_{a b}\right|=\left|\Delta_{a c}\right|+\left|\Delta_{c b}\right|$. Thus a two-particle BPS state $(a c)(c b)$ would have the same mass as a one-particle state $(a b)$, and can contribute to $C_{a b}$ in the same way. This means that $\mu_{a b}$ does not necessarily count the one-particle BPS doublets. In fact, $\mu_{a b}=1 / 2$ in the $q=1$ case, as was shown by explicit solution of the differential equation [5]. There is no such thing as a half-soliton, so in this case this contribution must arise solely from the two-particle states. It is important to note that even though two-particle states are not necessarily in a BPS representation, they can contribute to $C_{a b}$ [3]; the argument in the introduction applies only to one-particle states.

For $n=4 q$, the relations of the soliton numbers to the intersection numbers are essentially the same as in the case $n=4 q+2$ given in the last subsection. However, because of the collinearity, the definitions of $\mu_{i, i+q}$ and $\mu_{i, i-q}$ require special attention. Let us analyze this situation in the context of singularity theory. Consider three such critical values $W_{0}, W_{i}, W_{i+n / 4}$, with $t$ a non-critical point on the line between $W_{0}$ and $W_{i}$. As before, $\gamma_{i}$ and $\gamma_{0}$ are straight lines connecting $t$ to $W_{i}$ and $W_{0}$, but with $\gamma_{i+n / 4}$, we deform the line so that it makes a small semicircle around the origin. In $V_{t}$, the intersection between $\Delta_{0}$ and $\Delta_{i}$ gives $\mu_{0 i}$. In addition, $\Delta_{i}$ 
and $\Delta_{i+n / 4}$ intersect, but they may have common intersection points with $\Delta_{0} \circ \Delta_{i}$. The common intersection points give contributions which look like two-soliton states between vacua $X_{i}$ and $X_{i+n / 4}$ whose number we denote by $\mu_{i, i+n / 4}^{2}=\mu^{2}(n / 4)$, and the non-common intersection points give one-soliton states whose number is $\mu_{i, i+n / 4}^{1}=\mu^{1}(n / 4)$. The same analysis applies when considering the vacua $X_{i+n / 4}, X_{0}$ and $X_{i+n / 2}$. The vacua $X_{i}$ and $X_{i+n / 2}$ map into the same $W_{i}$, but to maintain the $\mathbb{Z}_{n}$-symmetry, the line between $W_{i}$ and $W_{i+n / 4}$ avoids the origin by a small semi-circle opposite to the above semi-circle. We include a minus sign in the definition of $\mu^{2}$ coming from the definitions of $\mu_{0 i}$ and $\mu_{0, i+n / 2}$, but not in the definition of $\mu^{1}$ since the straight lines are not exactly the same. Therefore, when $|i-j|=|j-k|=q$, with $1 \leq i, j \leq n / 2$ and $n / 2<k, l \leq n$, we define the soliton numbers as:

$$
\begin{aligned}
\Delta_{i} \circ \Delta_{j} & =\mu_{i j}^{1}+\mu_{i j}^{2} \\
\Delta_{k} \circ \Delta_{l} & =\mu_{k l}^{1}+\mu_{k l}^{2} \\
\Delta_{i} \circ \Delta_{k} & =\mu_{i k}^{1}-\mu_{i k}^{2}
\end{aligned}
$$

This procedure of separating the intersection matrix into two components is equivalent to a partial diagonalization of the intersection matrix. The Dynkin diagram of a singularity of a function of two variables can be often represented in the form of the Dynkin diagram of a real curve. The connection between the two Dynkin diagrams is done by a partial diagonalization of the intersection matrix. It is known [18] that the intersection numbers, after diagonalization, belong to the set of half-integer $\frac{1}{2} \mathbb{Z}$. We will indeed obtain intersection numbers of $1 / 2$ for three collinear vacua for which the above representation of Dynkin diagrams is valid.

We proceed now, as in the $n=4 q+2$ case, by expressing the intersections on invariant and anti-invariant spaces in terms of soliton numbers we need to compute. For the invariant space we have:

$$
\begin{aligned}
\widetilde{\Delta}_{i} \circ \widetilde{\Delta}_{i+p} & =2 \mu(p)+2 \mu(2 q-p) \equiv 2 \widetilde{\mu}(p) \\
\widetilde{\Delta}_{i} \circ \widetilde{\Delta}_{i+q} & =4 \mu^{2}(q) \equiv 2 \widetilde{\mu}(q)
\end{aligned}
$$

where $p=1 \ldots q-1$. There are again $q$ different $\widetilde{\mu}(i)$ in the invariant part. As in the case $n=4 q+2$, they are identified with the real soliton numbers for the superpotential $\widehat{W}(\widehat{X}, \widehat{Y})=$ $\frac{\widehat{X}^{(n+2) / 2}}{n+2}-\lambda \frac{\widehat{X}}{2}+\widehat{Y}^{2}$. Since these are known to be all -1 11, 5], we have

$$
\begin{aligned}
\mu(p)+\mu(2 q-p) & =-1, \\
\mu^{2}(q) & =-1 / 2
\end{aligned}
$$

The latter must be a two-soliton contribution, and we see how this contribution of $\pm 1 / 2$ to $\mu_{a b}$ arises naturally in singularity theory. We have thus proven directly that the behavior already observed for $q=1$ persists for all $q$.

Finding the soliton numbers in the anti-invariant space requires a new computation. The soliton numbers are related to the intersection numbers by

$$
\begin{gathered}
\widehat{\Delta}_{i} \circ \widehat{\Delta}_{i+p}=2 \mu(p)-2 \mu(2 q-p) \equiv 2 \widehat{\mu}(p), p=1, \ldots, q-1 \\
\widehat{\Delta}_{i} \circ \widehat{\Delta}_{i+q}=4 \mu^{1}(q) \equiv 2 \widehat{\mu}(q)
\end{gathered}
$$

Note that the one-soliton number given by $\mu^{1}(q)$ is in the anti-invariant space. To compute $\mu(0)$ and $\widehat{\mu}$ we use again equation (23). The vacua geometry in $W$-plane is represented in Figure 9 , and the sequence of points is:

$$
\begin{aligned}
k & =\{2 q, 2 q-1,2 q-2, \ldots \ldots, q+3, q+2, q+1\} \\
h & =\{q, q-1, q-2, \ldots ., 3,2,1\}
\end{aligned}
$$


where again $\operatorname{Im}(W(k)) \geq 0$, and $\operatorname{Im}(W(h)) \leq 0$. Let us first study the change of basis matrices

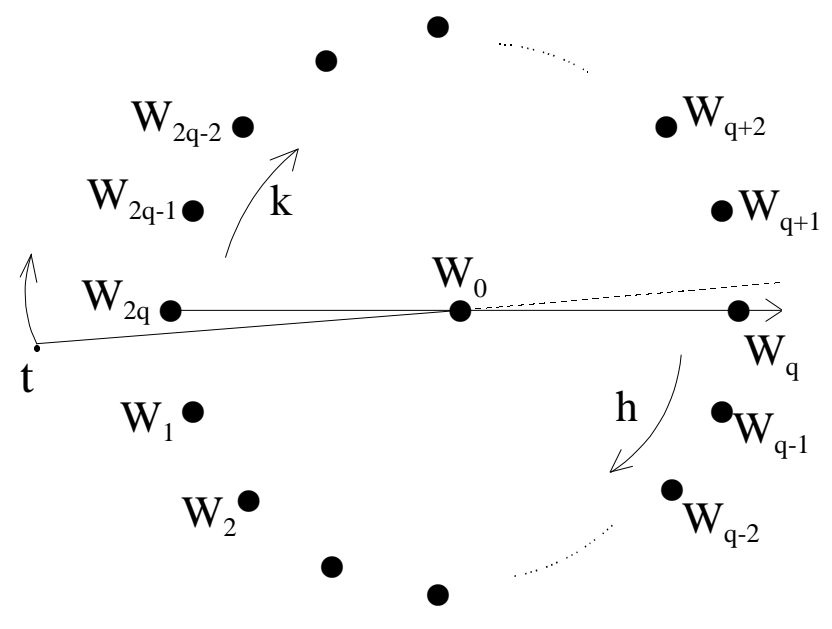

Figure 9: Vacua geometry in $W$-plane for $n=4 q$

associated with the collinear vacua $W_{0}, W_{q}$ and $W_{2 q}$. As $t$ moves, the straight line paths $\gamma_{0}, \gamma_{q}$ and $\gamma_{2 q}$ will change into $\gamma_{0}^{\prime}, \gamma_{q}^{\prime}$ and $\gamma_{2 q}^{\prime}$ as in Figure 10. It is known [18, 26] that two weakly distinguished bases can be obtained one from the other by iterations of operations $\alpha_{a}(b)$ and $\beta_{a}(b)$. There are two such change of paths operations to change $\gamma$ into $\gamma^{\prime}: \alpha_{0}(q) \alpha_{2 q}(q) \alpha_{2 q}(0)$ and $\alpha_{2 q}(0) \alpha_{2 q}(q) \alpha_{0}(q)$. However, they do not commute. Since we want to obtain soliton numbers

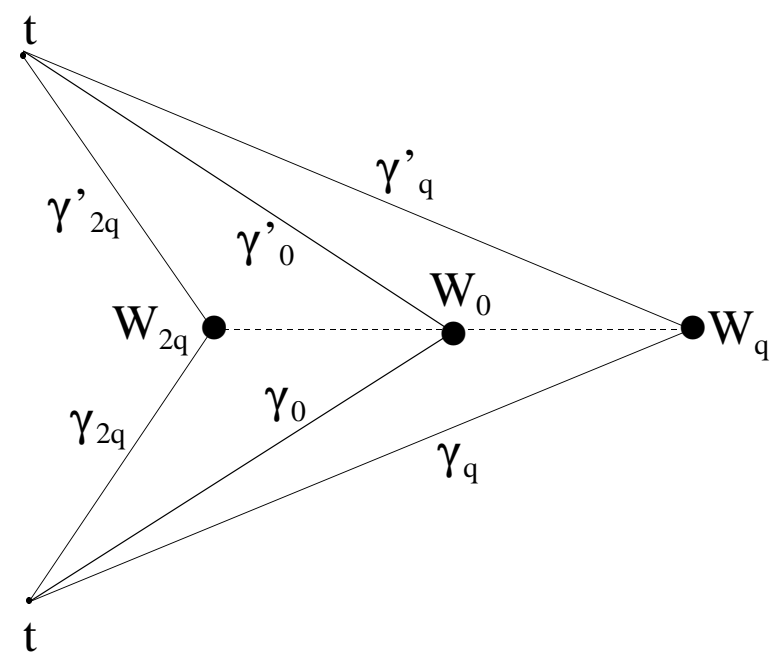

Figure 10: Three collinear vacua in $W$-plane: $W_{q}, W_{0}, W_{2 q}$

which do not depend on how the paths $\gamma$ transform into $\gamma^{\prime}$, we take the change of basis matrix as being the symmetrized change of basis matrices of the two operations. For example, for the above change of paths the change of basis is given by:

$$
M_{q, 0,2 q} \equiv \frac{1}{2}\left\{\left(I-\widehat{A}_{0,2 q}\right),\left(I-A_{q, 0}\right)\right\}\left(I-A_{q, 2 q}\right),
$$


where $A$ and $\widehat{A}$ have the same meaning as in the case $n=4 q+2$, and the anti-commutator has the elements:

$$
\begin{aligned}
\frac{1}{2}\left\{\left(I-\widehat{A}_{0,2 q}\right),\left(I-A_{q, 0}\right)\right\}_{i j} & \equiv \delta_{i j}-\left(\mu_{0}^{<1>}\right)_{i j}= \\
& =\delta_{i j}+\delta_{i, q} \delta_{j, 0} 2 \mu(0)-\delta_{i, 0} \delta_{j, 2 q} \mu(0)-\delta_{i, q} \delta_{j, 2 q} \mu^{2}(0)
\end{aligned}
$$

Such matrices $M$ can be connected in order as they appear in the following sense $(1 \leq i \leq q-1)$ :

$$
\begin{gathered}
R M_{q-i, 0,2 q-i} R^{-1}=M_{q-i+1,0,2 q-i+1} \\
R M_{2 q, 0, q} R^{-1}=-M_{1,0, q+1} \\
R M_{2 q-i, 0, q-i} R^{-1}=M_{2 q-i+1,0, q-i+1}
\end{gathered}
$$

The sets of lines which do not pass through the origin can also be connected in the same way. For a given direction the parallel lines contain half of the soliton numbers but each one appears twice. However, we can connect such parallel lines by using $R^{q}, R^{-q}$ to have each soliton number appearing once. Then, we obtain the monodromy matrix $M^{-}$:

$$
M^{-}=-\left[\left(I-\mu_{0}^{<1>}\right)\left(I-\mu_{2}^{<1>}\right) R^{q}\left(I+\mu_{1}^{<1>}\right)^{T} R^{q-1}\right]^{n / 2}
$$

where:

$$
\left(\mu_{2}^{<1>}\right)_{i j}=\delta_{i, q} \delta_{j, 2 q} \widehat{\mu}(q)+\left(\mu_{1}^{<1>}\right)_{i j}
$$

and $\mu_{1}^{<1>}$ is the sum of all $A$ matrices which correspond to the first and second set of parallel lines (excluding $A_{q, 2 q}$ ) such that all soliton numbers appear once. It has the elements:

$$
\begin{aligned}
\left(\mu_{1}^{<1>}\right)_{i j}= & \delta_{i, q} \delta_{j, 2 q-1} \widehat{\mu}(q-1)+\delta_{i, q+1} \delta_{j, 2 q-2} \widehat{\mu}(q-3)+\delta_{i, q+2} \delta_{j, 2 q-3} \widehat{\mu}(q-5)+\ldots \\
& +\delta_{i, q+1} \delta_{j, 2 q-1} \widehat{\mu}(q-2)+\delta_{i, q+2} \delta_{j, 2 q-2} \widehat{\mu}(q-4)+\delta_{i, q+3} \delta_{j, 2 q-3} \widehat{\mu}(q-6) \ldots
\end{aligned}
$$

Again, solving (23) reduces to solving:

$$
\operatorname{det}\left[\left(I-\mu_{0}^{<1>}\right)\left(I-\mu_{2}^{<1>}\right) R^{q}\left(I+\mu_{1}^{<1>}\right)^{T} R^{q-1}+z\right]=P^{-}(z)
$$

The computation of the determinant gives:

$$
\begin{aligned}
P^{-}(z)= & z^{2 q+1}-1-z^{2 q}(-1-\widehat{\mu}(1))+z(-1-\widehat{\mu}(1))+ \\
& +(-1)^{q} z^{q+1}\left(\mu^{2}(0)+\widehat{\mu}(q-1)+\widehat{\mu}(q)\right)+(-1)^{q-1} z^{q}\left(\mu^{2}(0)+\widehat{\mu}(q-1)-\widehat{\mu}(q)\right)+ \\
& +\sum_{k=1}^{q-2}\left[(-1)^{k+1} z^{2 q-k}(\widehat{\mu}(k)-\widehat{\mu}(k+1))+(-1)^{q-k-1} z^{q-k}(\widehat{\mu}(q-k-1)-\widehat{\mu}(q-k))\right] .
\end{aligned}
$$

Plugging in the polynomial from (23) gives us the solutions

$$
\begin{aligned}
\mu(0) & = \pm 1, \\
\widehat{\mu}(i) & =-1, \quad i=1, \ldots, q-1 \\
\widehat{\mu}(q) & =0
\end{aligned}
$$

which gives the original soliton numbers as

$$
\begin{aligned}
\mu^{1}(q) & =0, \\
\mu(p)-\mu(2 q-p) & =-1, \quad p=1, \ldots, q-1 .
\end{aligned}
$$


Finally, combining this with the results for invariant cycles $(26,27)$ yields

$$
\begin{gathered}
\mu(0)=1 \\
\mu(1)=\mu(2)=\mu(3)=\ldots=\mu\left(\left[\frac{n}{4}\right]-1\right)=1, \mu\left(\left[\frac{n}{4}\right]\right)=1 / 2 \\
\mu\left(\left[\frac{n}{4}\right]+1\right)=\mu\left(\left[\frac{n}{4}\right]+2\right)=\ldots=\mu\left(\left[\frac{n}{2}\right]\right)=0
\end{gathered}
$$

Note that there are no fundamental BPS doublets connecting vacua $i$ and $i+q$. because $\mu^{1}\left(\left[\frac{n}{4}\right]\right)$ is zero. As for $n$-odd, we display graphically the soliton numbers for several particular cases of $n$-even in figure 11 . In $W$ plane the symmetry is only $\mathbb{Z}_{n / 2}$. The pattern is clear: there is one line (one BPS doublet) connecting any two vacua except for the vacua which are collinear with the vacuum at the origin.

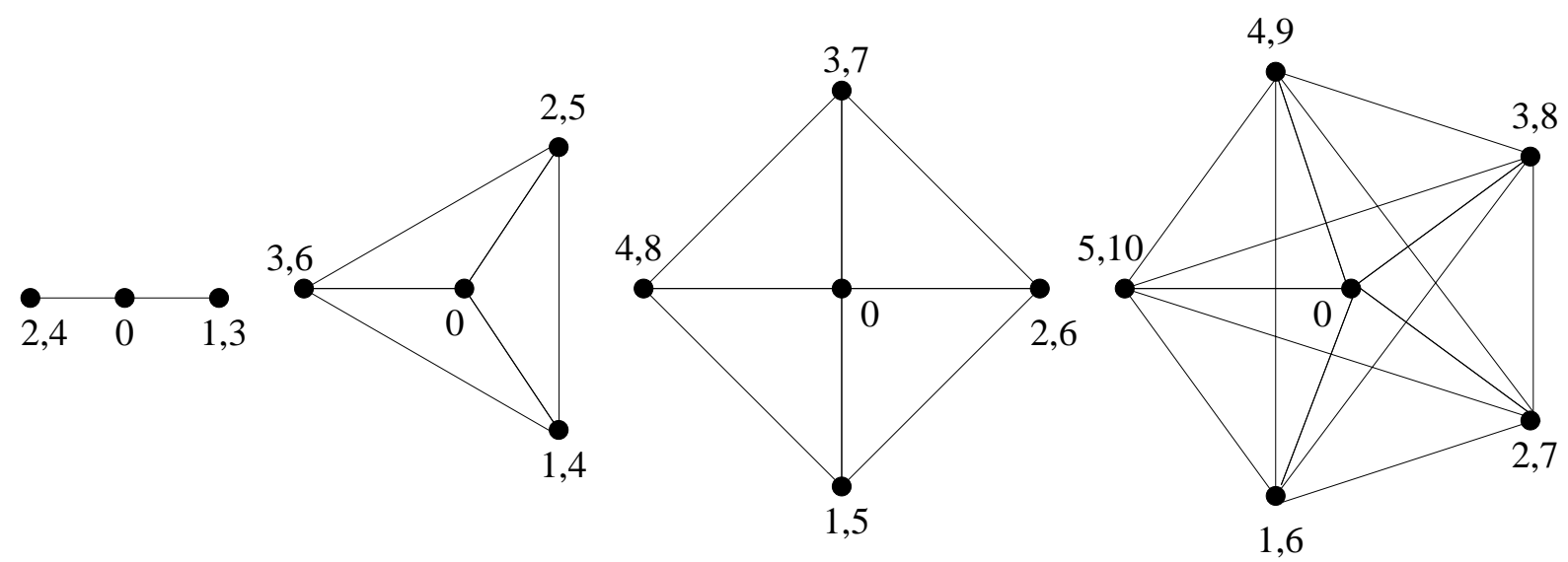

Figure 11: Solitons in the $W$-plane for $n=4,6,8,10$. Straight lines represent BPS states.

We end the computation for $n$ even by noticing the similarity between this case and the most relevant perturbation of the $D$ series of minimal superconformal models given by $W=$ $X^{(n+2) / 2}-X Y^{2}-\lambda X$. In fact, the two are related by an orbifold [13]. For this perturbed $D$ series one can do similar computations as in sections 5.1 and 5.2, and obtain exactly the same expressions for the determinants, and therefore the same soliton numbers.

\section{Non-BPS states}

We have found the full BPS spectrum of the $\mathcal{N}=(2,2)$ Landau-Ginzburg theory $X^{n+2}-\lambda X^{2}$ for any $n$. The Picard-Lefschetz theory of singularities of differentiable maps proved to be a powerful tool in finding the BPS spectrum exactly. This technique is more direct than using topological anti-topological fusion equations, because extracting the appropriate behavior from those equations explicitly is difficult at best.

There is one interesting consequence of this computation. As we already mentioned, the theories studied here are integrable [13, 14. Integrable models have the striking property that the $S$-matrix factorizes into two-body ones, and all scattering processes are completely elastic [27. This means that in any scattering process, the masses of the individual particles are conserved. The only possible consequences of scattering are a phase shift or changing internal quantum numbers. This fact, along with the fact that the boundary conditions at spatial infinity remain 


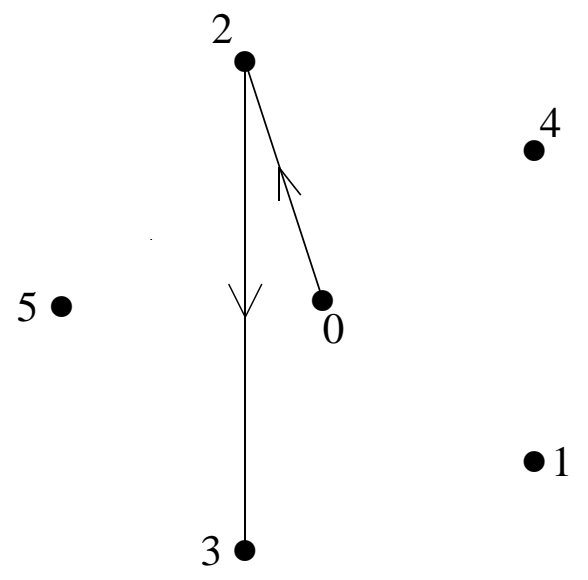

Figure 12: Two-soliton configuration in the $W$ plane when $n=5$

fixed in a collision, means that we can show there must be non-BPS single-particle states. The argument for this is quite simple. Consider a two-particle configuration $(0 a)(a b)$, where $a$ and $b$ are vacua other than the origin. The masses of these BPS particles are given by the length of the corresponding lines in the $W$ plane, namely $m_{0 a}=|W(a)-W(0)|$, and $m_{a b}=|W(b)-W(a)|$. Such a two-particle configuration is drawn for $n=7$ in figure 12. When these two particles scatter, they must scatter to two particles $(0 c)(c b)$. Since the theory is integrable, there can be no particle production, and we must have $m_{0 c}=m_{a b}$ and $m_{c b}=m_{0 a}$. Note that it is not possible for $c=a$; the solitons must change sides in the scattering event. In fact, in general there is no vacuum $c$ where $|W(c)-W(0)|=|W(b)-W(a)|$ or with $|W(b)-W(c)|=|W(a)-W(0)|$. This can be easily seen from the picture in figure 12: there is no line $(0 c)$ of the same length as the line (23), and no line (c3) of the same length as (02). This means that the final state in this scattering process cannot consist of BPS states! The theory must contain additional states, and the scattering of BPS states does not close onto itself.

We have therefore proven that there are non-BPS states mixing with the BPS states here, as opposed to the models discussed in [10, 11], where the entire spectrum consists only of BPS states. The simplest scenario consistent with integrability and the BPS spectrum is for there to exist non-BPS states (00). Presumably, these states are analogous to the breathers well known in the sine-Gordon field theory. Because of the integrability, the masses of these non-BPS quartets must be the same as the BPS states, except a non-BPS particle of mass $m_{0 i}$ is not required. We will give the $S$ matrix for all of these states in the sequel [12].

This work was supported in part by the Research Corporation under a Research Innovation Award, a DOE OJI award an NSF grant DMR-0104799, and a Sloan Foundation Fellowship.

\section{References}

[1] E. B. Bogomolny, Sov. J. Nucl. Phys. 24, 449 (1976); M. K. Prasad and C. M. Sommerfield, Phys. Rev. Lett. 35, 760 (1975). 
[2] N. Seiberg and E. Witten, Nucl. Phys. B 426, 19 (1994) [Erratum-ibid. B 430, 485 (1994)] arXiv:hep-th/9407087.

[3] S. Cecotti, P. Fendley, K. A. Intriligator and C. Vafa, Nucl. Phys. B 386, 405 (1992) arXiv:hep-th/9204102.

[4] S. Cecotti and C. Vafa, Nucl. Phys. B 367, 359 (1991).

[5] S. Cecotti and C. Vafa, Commun. Math. Phys. 158, 569 (1993) arXiv:hep-th/9211097.

[6] K. Hori and C. Vafa, arXiv:hep-th/0002222.

[7] A. Losev, M. A. Shifman and A. I. Vainshtein, Phys. Lett. B 522, 327 (2001) arXiv:hepth/0108153.

[8] J. Maldacena and L. Maoz, arXiv:hep-th/0207284

[9] P. Fendley, S. D. Mathur, C. Vafa and N. P. Warner, Phys. Lett. B 243, 257 (1990).

[10] P. Fendley and K. A. Intriligator, Nucl. Phys. B 372, 533 (1992) arXiv:hep-th/9111014.

[11] P. Fendley and K. A. Intriligator, Nucl. Phys. B 380, 265 (1992) arXiv:hep-th/9202011.

[12] A. Tirziu and P. Fendley, to appear.

[13] P. Fendley, W. Lerche, S. D. Mathur and N. P. Warner, Nucl. Phys. B 348, 66 (1991).

[14] P. Mathieu and M. A. Walton, Phys. Lett. B 254, 106 (1991).

[15] C. Gomez and G. Sierra, Nucl. Phys. B 419, 589 (1994) arXiv:hep-th/9312032.

[16] W. Lerche and N. P. Warner, Nucl. Phys. B 358, 571 (1991).

[17] V.I. Arnold, S.M. Gusein-Zade and A.N. Varčenko, Singularities of Differentiable Maps, Vol.I, Birkhäusser, Boston, 1988.

[18] V.I. Arnold, S.M. Gusein-Zade and A.N. Varčenko, Singularities of Differentiable Maps, Vol.II, Birkhäusser, Boston, 1988.

[19] E. Witten and D. Olive, Phys. Lett. B78, 97 (1978).

[20] E. Martinec, Phys. Lett. 217B, 431 (1989).

[21] C. Vafa and N. P. Warner, Phys. Lett. B 218, 51 (1989).

[22] R. Jackiw and C. Rebbi, Phys. Rev. D13, 3398 (1976).

[23] E. Witten, Nucl. Phys. B202, 253 (1982).

[24] V. I. Arnold, Russ. Math. Survey 33:5, 99 (1978)

[25] C.T.C. Wall, Bull. LMS 12, 169 (1980).

[26] S. P. Humphries, Quart. J. Math. Oxford 36, 215 (1985).

[27] A.B. Zamolodchikov and Al.B. Zamolodchikov, Ann. Phys. 120, 253 (1980). 